NASA/TM-2009-216166

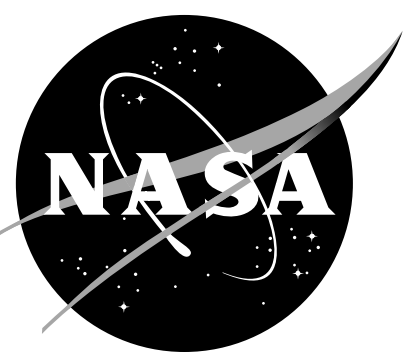

\title{
Boundary Closures For Fourth-order Energy Stable Weighted Essentially Non-Oscillatory Finite Difference Schemes
}

Travis C. Fisher

Purdue University, West Lafayette, Indiana

Mark H. Carpenter

Langley Research Center, Hampton, Virginia

Nail K. Yamaleev

North Carolina A\&T State University, Greensboro, North Carolina

Steven H. Frankel

Purdue University, West Lafayette, Indiana 


\section{NASA STI Program ... in Profile}

Since its founding, NASA has been dedicated to the advancement of aeronautics and space science. The NASA scientific and technical information (STI) program plays a key part in helping NASA maintain this important role.

The NASA STI Program operates under the auspices of the Agency Chief Information Officer. It collects, organizes, provides for archiving, and disseminates NASA's STI. The NASA STI Program provides access to the NASA Aeronautics and Space Database and its public interface, the NASA Technical Report Server, thus providing one of the largest collection of aeronautical and space science STI in the world. Results are published in both non-NASA channels and by NASA in the NASA STI Report Series, which includes the following report types:

- TECHNICAL PUBLICATION. Reports of completed research or a major significant phase of research that present the results of NASA programs and include extensive data or theoretical analysis. Includes compilations of significant scientific and technical data and information deemed to be of continuing reference value. NASA counterpart of peer-reviewed formal professional papers, but having less stringent limitations on manuscript length and extent of graphic presentations.

- TECHNICAL MEMORANDUM. Scientific and technical findings that are preliminary or of specialized interest, e.g., quick release reports, working papers, and bibliographies that contain minimal annotation. Does not contain extensive analysis.

- CONTRACTOR REPORT. Scientific and technical findings by NASA-sponsored contractors and grantees.
- CONFERENCE PUBLICATION. Collected papers from scientific and technical conferences, symposia, seminars, or other meetings sponsored or co-sponsored by NASA.

- SPECIAL PUBLICATION. Scientific, technical, or historical information from NASA programs, projects, and missions, often concerned with subjects having substantial public interest.

- TECHNICAL TRANSLATION. Englishlanguage translations of foreign scientific and technical material pertinent to NASA's mission.

Specialized services also include creating custom thesauri, building customized databases, and organizing and publishing research results.

For more information about the NASA STI Program, see the following:

- Access the NASA STI program home page at http://www.sti.nasa.gov

- E-mail your question via the Internet to help@sti.nasa.gov

- Fax your question to the NASA STI Help Desk at 443-757-5803

- Phone the NASA STI Help Desk at 443-757-5802

- Write to: NASA STI Help Desk NASA Center for AeroSpace Information 7115 Standard Drive Hanover, MD 21076-1320 
NASA/TM-2009-216166

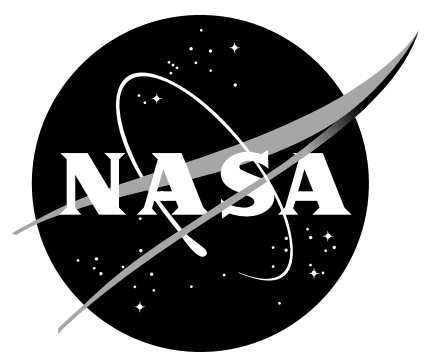

\section{Boundary Closures For Fourth-order Energy Stable Weighted Essentially Non-Oscillatory Finite Difference Schemes}

Travis C. Fisher

Purdue University, West Lafayette, Indiana

Mark H. Carpenter

Langley Research Center, Hampton, Virginia

Nail K. Yamaleev

North Carolina A $6 T$ State University, Greensboro, North Carolina

Steven H. Frankel

Purdue University, West Lafayette, Indiana 


\section{Acknowledgments}

Work performed in partial fulfillment of LaRC LARSS summer fellowship program, Technical monitor Robert Baurle. Special thanks are extended to the faculty and staff of Technical University of Delft, Delft, The Netherlands, for many stimulating discussions on this and other topics.

The use of trademarks or names of manufacturers in this report is for accurate reporting and does not constitute an offical endorsement, either expressed or implied, of such products or manufacturers by the National Aeronautics and Space Administration.

Available from:

NASA Center for AeroSpace Information 7115 Standard Drive

Hanover, MD 21076-1320

443-757-5802 


\begin{abstract}
A general strategy exists for constructing Energy Stable Weighted Essentially NonOscillatory (ESWENO) finite difference schemes up to eighth-order on periodic domains. These ESWENO schemes satisfy an energy norm stability proof for both continuous and discontinuous solutions of systems of linear hyperbolic equations. Herein, boundary closures are developed for the fourth-order ESWENO scheme that maintain wherever possible the WENO stencil biasing properties, while satisfying the summation-by-parts (SBP) operator convention, thereby ensuring stability in an $L_{2}$ norm. Second-order, and third-order boundary closures are developed that achieve stability in diagonal and block norms, respectively. The global accuracy for the second-order closures is three, and for the third-order closures is four. A novel set of non-uniform flux interpolation points is necessary near the boundaries to simultaneously achieve 1) accuracy, 2) the SBP convention, and 3) WENO stencil biasing mechanics.
\end{abstract}

\title{
Contents
}

1 Introduction $\quad 2$

2 Definitions $\quad 4$

2.1 Complementary Grids . . . . . . . . . . . . . . . . . . . . 4

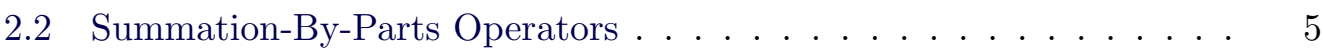

2.2.1 Central Fourth-order SBP Operators . . . . . . . . . . 6

3 Expressing Summation-By-Parts Operators in Flux Form $\quad \mathbf{8}$

3.1 General Structure of ESWENO Schemes . . . . . . . . . . . 8

3.2 Nonuniform Flux-Points . . . . . . . . . . . . . . . . . . 9 9

3.3 Interpolation Operators and Preferred Weights . . . . . . . . . . . 11

4 Energy Stabilization Terms $\quad 11$

5 Numerical Tests $\quad \mathbf{1 2}$

5.1 Eigenvalues of the ESWENO operator . . . . . . . . . . . . 13

5.2 Linear Wave Equation . . . . . . . . . . . . . . . . . . . . . 14

5.2 .1 Sine Wave . . . . . . . . . . . . . . . . . . . . . . . . . 14

5.2 .2 Square Wave . . . . . . . . . . . . . . . . . . 14

5.3 Euler Equations . . . . . . . . . . . . . . . . . . . . . . 15

5.3.1 Subsonic One-Dimensional Nozzle . . . . . . . . . . . . 17

5.3.2 Transonic One-Dimensional Nozzle . . . . . . . . . . . . 17

5.3.3 Two Dimensional Inviscid Vortex . . . . . . . . . . . . . . 18

5.4 Supersonic Shear Layers . . . . . . . . . . . . . . . . . . . . . . . . 19

6 Conclusions 21

$\begin{array}{lr}\text { A Smoothness Indicators } & \mathbf{2 3}\end{array}$ 
C Fourth-order Diagonal-Norm Operator: $D_{\mathbf{2}-4-2} \quad 27$

C.1 Differentiation Matrix . . . . . . . . . . . . . . . . 27

C.2 Interpolation Operators . . . . . . . . . . . . . . . . . . . . . . . . . . . . 28

C.3 Target Weights . . . . . . . . . . . . . . . . 28

C.4 Energy Stable Terms . . . . . . . . . . . . . . . . . . 29

D Fourth-order Block-Norm Operator: $D_{3-4-3} \quad 31$

D.1 Differentiation Matrix . . . . . . . . . . . . . . 31

D.2 Interpolation Operators . . . . . . . . . . . . . . . . 32

D.3 Target Weights . . . . . . . . . . . . . . . . . . . . . . . . . . . . . . . . . . . 32

D.4 Energy Stable Terms . . . . . . . . . . . . . . . 33

E References $\quad 34$

\section{Introduction}

High order Weighted Essentially Non-Oscillatory (WENO) methods are ideally suited for high fidelity simulations of complex physics containing discontinuities. An example of a typical application is a canonical simulation of sound generated by a shock-vortex interaction $[1,2]$. The high-order nature of WENO efficiently resolves the subtle details of the sound generation and propagation, while the stencil biasing mechanics ensures robust, high resolution properties in the vicinity of the shock.

Although ideal for infinite or periodic domains, conventional WENO formulations encounter serious challenges at nodes that are near domain boundaries. To illustrate this, first realize that all high-order finite-difference (HOFD) formulations use "inward biased" stencils near boundaries that maintain the accuracy and stability of the interior scheme. Next, note that WENO schemes invoke stencil biasing mechanics throughout the entire domain, including nodes adjoining the boundaries (e.g. fourth-order WENO schemes test three candidate stencils at each node). Thus, not only must the boundary closures used for WENO schemes be 1) inward-biased, 2) stable and 3) accurate, they must also be stable for any possible combination of candidate stencils occurring anywhere in the domain. Simultaneously satisfying these constraints is a remote possibility if left strictly to chance.

Many ad hoc boundary closures have been proposed for conventional WENO schemes, but most implementations still rely on low order boundary closures to achieve stability and robustness. For flow near a wall, Shen et al. [3] constructed the domain such that the no slip condition is enforced at the WENO flux point. Pressure was extrapolated to the wall using a third order scheme, and the next WENO flux cell was treated with a third order reconstruction. This procedure reduces the global order of accuracy of their 5th order WENO scheme. Alternatively, ghost points can be used in combination with physical boundary conditions to specify data in stencils that extend outside of the domain [4]. This reduces the generality of the boundary 
treatment. Another strategy is to simply discard stencils that extend outside of the domain [4].

The principle objective of this work is to develop boundary closures to complement the periodic domain Energy Stable WENO finite difference methodology developed and reported in Refs. [5] and [6]. The new interior/boundary ESWENO schemes (henceforth referred to as "finite-domain ESWENO") retain all the salient features of the original periodic schemes, including: 1) conservation and $L_{2}$-energy stability for constant coefficient (linear) hyperbolic systems, including those with discontinuous initial or boundary data, 2) design order accuracy throughout the entire domain, especially regions near the boundaries or near smooth extrema, and 3) full stencil biasing mechanics (Left, Central, Right) at all possible points.

The finite-domain ESWENO schemes are constructed by adding a "special" nonlinear artificial dissipation term to a conventional WENO scheme (e.g. those found in Refs. $[7,8]$ ). The additional term is design-order accurate for smooth solutions, including smooth extrema, and is constructed such that the resulting ESWENO scheme is stable in the $L_{2}$-energy norm for both continuous and discontinuous solutions. ${ }^{1}$ In this initial work, we focus exclusively on the central fourth-order class of ESWENO schemes, leaving the development of 6th- and 8th-order schemes to a later date.

The strategy for developing finite-domain ESWENO schemes is for the most part equivalent to that used to build the periodic domain ESWENO schemes. The essential elements are summarized as follows:

- Develop a 4th-order finite-domain target scheme that is stable, conservative and accurate for smooth flows. This task is accomplished using the summationby-parts (SBP) [6,9] framework (as was done in the periodic case). The SBP framework requires the construction of accurate discrete schemes that satisfy basic matrix conditions, that if met guarantee discrete stability and conservation.

- The target scheme is recast into the "dual-grid" framework of the conventional WENO approach, whereby the solution is stored/advanced at the gridpoints, while interface fluxes are constructed at the "half-points".

- The stability of the resulting stencil is tested, and if unstable, is modified with a special artificial dissipation term. This ensures that the discrete eigenvalues of the spatial scheme are bounded within the left-half of the complex plane.

The organization of the paper is as follows. Section 2 introduces a dual-grid nomenclature and summarizes the necessary SBP formulas including a discrete stability proof. Section 3 summarizes the implementation of the ESWENO scheme, and then expresses the baseline ESWENO operator in SBP form. Section 4 formulates the artificial dissipation term necessary for the ESWENO approach. Section

\footnotetext{
${ }^{1}$ The conventional WENO scheme, does not have such an energy estimate. Thus, while the spectrum of the symmetric part of the ESWENO scheme is always located in the left half of the complex plane, the symmetric part of the WENO scheme could have positive eigenvalues.
} 
5 presents a summary of numerical test cases. Conclusions and directions are presented in Section 6. An appendix is included with all the implementation details.

\section{Definitions}

\subsection{Complementary Grids}

The implementation of the ESWENO (WENO) stencil biasing mechanics, as a matter of convenience, uses two complementary sets of gridpoints, differing in dimension by one. As such, two discrete sets of points

$$
\mathbf{x}_{N}=\left[x_{1}, x_{2}, \cdots, x_{N-1}, x_{N}\right]^{T} \quad ; \quad \overline{\mathbf{x}}_{M}=\left[\bar{x}_{0}, \bar{x}_{1}, \cdots, \bar{x}_{N-1}, \bar{x}_{N}\right]^{T}
$$

are defined on the interval $0 \leq x \leq 1$. Furthermore, a projection is defined of any function $\phi$ onto $\mathbf{x}_{N}$ and $\overline{\mathbf{x}}_{M}$ respectively as

$$
\phi=\left[\phi\left(x_{1}\right), \phi\left(x_{2}\right), \cdots, \phi\left(x_{N-1}\right), \phi\left(x_{N}\right)\right]^{T} \quad ; \quad \bar{\phi}=\left[\phi\left(\bar{x}_{0}\right), \phi\left(\bar{x}_{1}\right), \cdots, \phi\left(\bar{x}_{N-1}\right), \phi\left(\bar{x}_{N}\right)\right]^{T}
$$

The $\mathbf{x}_{N}$ is a uniform set of "solution points" and stores the discrete solution $\mathbf{u}$. Surrounding each solution point, $\mathbf{x}_{N j}$, a control volume is constructed, whereby the governing conservation laws are enforced. A second set of "flux points", $\overline{\mathbf{x}}_{M}$, is situated at the interfaces between adjoining control volumes, where data interpolated from $\mathbf{u}$ is used to construct interface fluxes $\bar{f}$ and establish a simple flux balance.

Figure (1) illustrates the relationship between the solution-mesh $\mathbf{x}_{N}$ and the flux-mesh $\overline{\mathbf{x}}_{M}$.

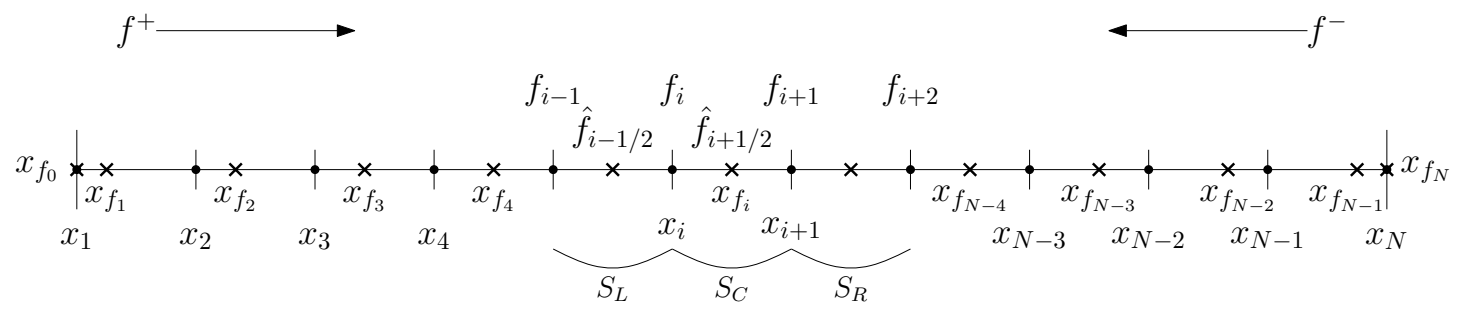

Figure 1. The computational domain is depicted. The ESWENO solution and flux points, and the smoothness indicator stencils $S^{L}, S^{C}$ and $S^{R}$ are shown.

The biasing mechanics move data via interpolation or differentiation between the two sets of points using non-square matrix operations. For example, a flux constructed on the high-dimensional set from data on the low-dimensional set, is represented as

$$
\overline{\mathbf{f}}={ }_{M} \mathcal{I}_{N} \mathbf{f}
$$

where $M=N+1$ and $M \mathcal{I}_{N}$ is an $[(N+1) \times N]$ matrix. 


\subsection{Summation-By-Parts Operators}

Consider a linear, scalar wave equation

$$
\begin{aligned}
& \frac{\partial u}{\partial t}+\frac{\partial f}{\partial x}=0, \quad f=a u, \quad t \geq 0, \quad 0 \leq x \leq 1, \\
& u(x, 0)=u_{0}(x) ; u(0, t)=g(t)
\end{aligned}
$$

where $u$ is the continuous solution, $a$ is a constant and $u_{0}(x)$ is a bounded piecewise continuous function. Without loss of generality, assume that $a \geq 0$ on the interval $0 \leq x \leq 1$. Applying the energy method to equation (2) leads to

$$
\frac{d}{d t}\|u\|_{L_{2}}^{2}+a\left[u^{2}(1, t)-g^{2}(t)\right]=0
$$

where $\|\cdot\|_{L_{2}}$ is the continuous $L_{2}$ norm. Thus, equation (2) admits an energy solution that is strictly dissipative (if $g(t)=0, a \geq 0$ ) for all time. We now develop using mimetic techniques (see Ref. [6] or Ref. [10]) a class of discrete spatial operators for which the discrete energy is bounded from above by the continuous target estimate provided by equations (2) and (3).

Define the low dimensional space $\mathbf{x}_{N}$ to be a uniform grid $x_{j+1}=j \Delta x, j=$ $0, \cdots, N-1$, with $\Delta x=1 /(N-1)$. On this grid, we define a flux $\mathbf{f}=a \mathbf{u}$ and its derivative $\mathbf{f}_{x}=a \mathbf{u}_{x}$, where $\mathbf{u}=\left[u\left(x_{1}, t\right), \ldots, u\left(x_{N}, t\right)\right]^{T}$ and $\mathbf{u}_{x}=\left[u_{x}\left(x_{1}, t\right), \ldots\right.$, $\left.u_{x}\left(x_{N}, t\right)\right]^{T}$ are projections of the continuous solution $u$ and its derivative $u_{x}$ onto the computational grid $\mathbf{x}_{N}$. Next, define a fourth-order approximation for the first-order derivative term in equation (2) as

$$
\frac{\partial \mathbf{f}}{\partial x}=D \mathbf{f}+O\left(\Delta x^{4}\right)
$$

Placing a mild restriction on the generality of the derivative operator (see Ref. [10] or Ref. [6]), the matrix $D$ is expressed in the following form:

$$
\begin{array}{rll}
D=\mathcal{P}^{-1}[\mathcal{Q}+\mathcal{R}] & ; \quad \mathcal{Q}+\mathcal{Q}^{T}=\operatorname{Diag}[-1,0, \cdots, 0,1] \\
\mathcal{R}=\mathcal{R}^{T} & ; \quad \mathbf{v}^{T} \mathcal{R} \mathbf{v} \geq 0 \\
\mathcal{P}=\mathcal{P}^{T} & ; \quad \mathbf{v}^{T} \mathcal{P} \mathbf{v}>0
\end{array}
$$

for any real vector $\mathbf{v} \neq \mathbf{0}$. The matrix $\mathcal{R}$ is defined by the expression

$$
\mathcal{R}=\Lambda_{0}+D_{1} \Lambda_{1}\left[D_{1}\right]^{T}+D_{1}\left[D_{1}\right]^{T} \Lambda_{2} D_{1}\left[D_{1}\right]^{T}+D_{1}\left[D_{1}\right]^{T} D_{1} \Lambda_{3}\left[D_{1}\right]^{T} D_{1}\left[D_{1}\right]^{T}
$$

which allows a constructive means of forming a symmetric semi-definite discrete

operator. Herein we define the matrices $\Lambda_{i}$ to be diagonal positive semidefinite matrices of the appropriate size: i.e.

$$
\begin{gathered}
\Lambda_{e}=\operatorname{Diag}\left[\lambda_{1}, \cdots, \lambda_{N}\right] \quad ; \quad \lambda_{j} \geq 0, j=1, N \quad ; \quad e=2 p, p=0,1 \\
\Lambda_{o}=\operatorname{Diag}\left[\lambda_{0}, \cdots, \lambda_{N}\right] \quad ; \quad \lambda_{j} \geq 0, j=0, N \quad ; \quad o=2 p+1, p=0,1
\end{gathered}
$$


Using the definitions of the SBP operators [eqs. (4)-(6)], and defining on $\mathbf{x}_{N}$ the discrete approximate solution $\mathbf{u}$ and flux $\mathbf{f}=a \mathbf{u}$, the semi-discrete counterpart of equation (2) becomes

$$
\begin{gathered}
\frac{\partial \mathbf{u}}{\partial t}+\mathcal{P}^{-1} \mathcal{Q} \mathbf{f}= \\
-\mathcal{P}^{-1}\left[\Lambda_{0}+D_{1} \Lambda_{1}\left[D_{1}\right]^{T}+D_{1}\left[D_{1}\right]^{T} \Lambda_{2} D_{1}\left[D_{1}\right]^{T}+D_{1}\left[D_{1}\right]^{T} D_{1} \Lambda_{3}\left[D_{1}\right]^{T} D_{1}\left[D_{1}\right]^{T}\right] \mathbf{f}
\end{gathered}
$$

To show that the above finite-difference scheme is stable, the energy method is used. Multiplying equation (7) with $\mathbf{u}^{T} P$, adding the result to its transpose, and rearranging the expression using equation (5), yields the semi-discrete energy equation $^{2}$

$$
\frac{d}{d t}\|\mathbf{u}\|_{\mathcal{P}}^{2}+a\left[u^{2}(N, t)-g^{2}(t)\right]=-2 a \sum_{i=0}^{q}\left(\left[D_{1}{ }^{i}\right]^{T} \mathbf{u}\right)^{T} \Lambda_{i}\left[D_{1}{ }^{i}\right]^{T} \mathbf{u} \leq 0
$$

The right-hand side of equation (8) is non-positive because the diagonal matrix $\Lambda_{n}$ is positive semidefinite $\left[\mathbf{v}^{T} \Lambda_{n} \mathbf{v} \geq 0\right.$ for all real $\mathbf{v}$ of length $\left.N\right]$ and $a \geq 0$; thus the stability of the finite-difference scheme given by equation (7) is assured. This result can be summarized in the following theorem:

Theorem 1 The approximation [eq. (7)] of the problem [eq. (2)] is stable if equations [(4)-(6)] hold.

Remark. The finite-difference scheme [eq. (7)] constructed as an approximation of equation (2) is inherently nonlinear, despite the linearity of (2). Indeed, the matrices $\mathcal{Q}$ and $\Lambda_{n}$ are nonlinear matrices [i.e., $\mathcal{Q}=\mathcal{Q}(\mathbf{u})$ and $\Lambda_{n}=\Lambda_{n}(\mathbf{u})$ ].

Remark. Although the matrices $\mathcal{Q}$ and $\Lambda_{n}$ are constrained in form by $\mathcal{Q}+\mathcal{Q}^{T}=$ $\operatorname{Diag}[-1,0, \cdots, 0,1]$ and $\mathbf{v}^{T} \Lambda_{n} \mathbf{v} \geq 0$, no specific assumptions are made about the values of the coefficients.

Remark. All matrices present in equation (7) need not be square. Indeed, it is advantageous to define the $[N \times(N+1)]$ dimensional matrix $D_{1}$ to be

$$
D_{1}=\left(\begin{array}{cccccc}
-1 & 1 & 0 & 0 & 0 & 0 \\
0 & -1 & 1 & 0 & 0 & 0 \\
0 & 0 & \ddots & \ddots & 0 & 0 \\
0 & 0 & 0 & -1 & 1 & 0 \\
0 & 0 & 0 & 0 & -1 & 1
\end{array}\right)
$$

to properly account for the difference between the dimension of the $\mathbf{x}_{N}$ and $\overline{\mathbf{x}}_{M}$ grids.

\subsubsection{Central Fourth-order SBP Operators}

The target operator used herein for the ESWENO scheme is a fourth-order nondissipative (i.e. $\mathcal{R}=0$ ) central SBP operator, for which equation (5) reduces

\footnotetext{
${ }^{2}$ The nomenclature $D_{1}{ }^{i}, i=1, q$ used in equation (8) is not valid for nonsquare matrices. Herein, define $D_{1}^{2 p}$ to mean $\left\{D_{1}\left[D_{1}\right]^{T}\right\}^{p} ; p=1, q$.
} 
to $D=\mathcal{P}^{-1} \mathcal{Q}$ subject to the constraints $\mathcal{Q}+\mathcal{Q}^{T}=\operatorname{Diag}[-1,0, \cdots, 0,1]$ and $\mathcal{P}=\mathcal{P}^{T} ; \mathbf{v}^{T} \mathcal{P} \mathbf{v}>0$.

The matrices $\mathcal{P}$ and $\mathcal{Q}$ are composed of the conventional banded operators in the interior of the domain:

$$
P_{I}=\Delta x I \quad ; \quad Q_{I}=\text { Pentadiagonal }\left[\frac{1}{12}, \frac{-8}{12}, 0, \frac{8}{12}, \frac{-1}{12}\right] .
$$

Boundary closures satisfying the SBP convention require auxiliary stencils at $N_{b}$ points near each boundary. Elsewhere, it is shown [11] that $N_{b} \geq 4$ is required to satisfy the necessary accuracy, symmetry and skew-symmetry constraints. Herein, the $N_{b}=4$ is assumed, whereby the matrices $\mathcal{P}$ and $\mathcal{Q}$ take the form

$$
\mathcal{P}=\Delta x\left(\begin{array}{ccc}
P_{0} & 0 & 0 \\
0 & I & 0 \\
0 & 0 & \left(P_{0}\right)^{P T}
\end{array}\right) \quad ; \quad \mathcal{Q}=\left(\begin{array}{ccc}
Q_{0} & Q_{d} & 0 \\
-Q_{d}^{T} & Q_{I} & Q_{d} \\
0 & -Q_{d}^{T} & -\left(Q_{0}\right)^{P T}
\end{array}\right)
$$

with

$$
\begin{gathered}
P_{0}=\left(\begin{array}{llll}
p_{11} & p_{12} & p_{13} & p_{14} \\
p_{12} & p_{22} & p_{23} & p_{24} \\
p_{13} & p_{23} & p_{33} & p_{34} \\
p_{14} & p_{24} & p_{34} & p_{44}
\end{array}\right) ; Q_{0}=\left(\begin{array}{cccc}
\frac{-1}{2} & q_{12} & q_{13} & q_{14} \\
-q_{12} & 0 & q_{23} & q_{24} \\
-q_{13} & -q_{23} & 0 & q_{34} \\
-q_{14} & -q_{24} & -q_{34} & 0
\end{array}\right) ; \\
Q_{d}=\left(\begin{array}{cccc}
0 & \ldots & & \\
0 & \ldots & & \\
\frac{-1}{12} & 0 & \ldots & \\
\frac{8}{12} & \frac{-1}{12} & 0 & \ldots
\end{array}\right)
\end{gathered}
$$

where $P T$ denotes the per-symmetric transpose.

Two types of $\mathcal{P}$-norms are typically used to prove stability with SBP discretizations: diagonal, and block. The diagonal-norm is advantageous when considering equations of more complexity than the constant coefficient, linear equation considered herein. Indeed, SBP proofs using the diagonal-norm extend to variablecoefficient problems [12], as well as certain nonlinear formulations [13,14], a property not shared by the block-norm boundary closures. Diagonal-norm boundary closures can not preserve the full design-order potential of the interior operator. Closures satisfying the matrix constraints in equation (8) with a fourth-order interior scheme, can have accuracy no higher than second-order. Thus, the global accuracy of this combination is only third-order $[15,16]$, or in general $p+1$-order despite $2 p$-order accuracy in the interior. (The nomenclature $p-2 p-p ; p=1, \cdots$ is sometimes used to describe the diagonal-norm formulations. See Ref. [9] and Ref. [11] for further discussion.)

Although block-norm closures are less robust than their diagonal-norm counterparts, for variable-coefficient linear or nonlinear equations, they enable the use of $2 p-1$-order stencils, and preserve the formal accuracy of the $2 p$-order interior operator. For example, third-order boundary closures preserve the formal accuracy of the fourth-order interior scheme. Furthermore, the additional parameters in the 
$\mathcal{P}$-norm (six, in the $(4 \times 4)$ case) can be used to optimize other properties of the third-order closure. Indeed, two free parameters (herein denoted $\vartheta_{l}, l=1,2$ ) remain, after satisfying all accuracy, and SBP matrix constraints, when using a $(4 \times 4)$ block norm to close a fourth-order interior. Both parameters, as will be shown in the next section, are needed to develop stable, and accurate fourth-order ESWENO boundary closures.

\section{Expressing Summation-By-Parts Operators in Flux Form}

The next objective is to recast the target fourth-order central SBP operator $(D=$ $\mathcal{P}^{-1} \mathcal{Q}$ defined on the $\mathbf{x}_{N}$ grid) into the "dual-grid" framework of the conventional ESWENO/WENO approach. Of particular interest is extending the ESWENO's "interior" stencil biasing mechanics, to those stencils at or near domain boundaries. We begin with a cursory description of the fourth-order ESWENO scheme, followed by its representation in matrix form.

\subsection{General Structure of ESWENO Schemes}

The conventional high-order WENO/ESWENO finite difference scheme for the scalar 1-D wave equation (Eq. (2)) can be written component-wise in the following semidiscrete form:

$$
\frac{d u_{j}}{d t}+\frac{\bar{f}_{j+\frac{1}{2}}-\bar{f}_{j-\frac{1}{2}}}{\Delta \bar{x}_{M_{j}}}=0
$$

The numerical flux $\bar{f}_{j+\frac{1}{2}}$ for the central fourth-order WENO scheme is given by the expression

$$
\bar{f}_{j+\frac{1}{2}}=\bar{w}_{j+1 / 2}^{L} \bar{f}_{j+1 / 2}^{L}+\bar{w}_{j+1 / 2}^{C} \bar{f}_{j+1 / 2}^{C}+\bar{w}_{j+1 / 2}^{R} \bar{f}_{j+1 / 2}^{R},
$$

where $\overline{\mathbf{f}}^{(r)}={ }_{M} \mathcal{I}_{N}^{(r)} \mathbf{f}, r=\{L, C, R\}$ are second order fluxes constructed from data interpolated using three distinct stencils $S^{L}, S^{C}$, and $S^{R}$.

The nonlinear weight functions $\bar{w}_{j+1 / 2}^{(r)}, r=\{L, C, R\}$ used in equation (13), are constructed such that the interface flux $\bar{f}_{j+\frac{1}{2}}$ is a convex combination of the three candidate second-order fluxes. The weight functions are constructed using the expressions

$$
\bar{w}_{j+1 / 2}^{(r)}=\frac{\bar{\alpha}_{j+1 / 2}^{(r)}}{\sum_{r} \bar{\alpha}_{j+1 / 2}^{(r)}} \quad ; \quad \bar{\alpha}_{j+1 / 2}^{(r)}=\bar{d}_{j+1 / 2}^{(r)}\left(1+\frac{\bar{\tau}_{j+1 / 2}}{\epsilon+\bar{\beta}_{j+1 / 2}^{(r)}}\right), \quad r=L, C, R
$$

\footnotetext{
${ }^{3}$ Figure (1) shows a schematic of the interpolation process. Each second-order flux is constructed from data obtained using only two points: $S^{L}=\left\{x_{j-1}, x_{j}\right\}, S^{C}=\left\{x_{j}, x_{j+1}\right\}$, and $S^{R}=\left\{x_{j+1}, x_{j+2}\right\}$. The $r=\{L, C, R\}$ nomenclature identifies the origin of the data relative to the interface position. The interpolates need not be limited to three; indeed $S^{L L}=\left\{x_{j-2}, x_{j-1}\right\}$, and $S^{R R}=\left\{x_{j+2}, x_{j+3}\right\}$ can be included near boundaries to increase the fidelity of the boundary closures.
} 
where $\bar{d}_{j+1 / 2}^{(r)}$ is the target weight of the candidate stencil. If no stencil biasing occurs (a well resolved solution), the target central difference operators are recovered, and the functions $\bar{w}^{(r)}$ used as weights in equation (13) reduce to

$$
\bar{\omega}_{j+1 / 2}^{(r)}=\frac{\bar{d}_{j+1 / 2}^{(r)}}{\sum_{r} \bar{d}_{j+1 / 2}^{(r)}}=\bar{d}_{j+1 / 2}^{(r)} .
$$

Definitions of the parameters $\bar{\tau}_{j+1 / 2}, \epsilon, \bar{\beta}_{j+1 / 2}^{(r)}, r=L, C, R$, as well as implementation details are included in the appendix.

The flux derivative expressed in equation (12) in component form can be written as

$$
\frac{\bar{f}_{j+\frac{1}{2}}-\bar{f}_{j-\frac{1}{2}}}{\Delta \bar{x}_{M_{j}}} \rightarrow\left(\left[\delta \overline{\mathbf{x}}_{M}\right]^{-1} D_{1} \overline{\mathbf{f}}\right)_{j}
$$

where $\left[\delta \overline{\mathbf{x}}_{M}\right]$ is the diagonal matrix $\left[\delta \overline{\mathbf{x}}_{M}\right]=\operatorname{Diag}\left[D_{1} \overline{\mathbf{x}}_{M}\right]$ that accounts for variable cell sizes. Further expanding the flux function $\overline{\mathbf{f}}$ in equation (15) in terms of matrix interpolation operators, and equating the result with the target SBP form, yields the expression

$$
\left[\delta \overline{\mathbf{x}}_{M}\right]^{-1} D_{1} \overline{\mathbf{f}}=\left[\delta \overline{\mathbf{x}}_{M}\right]^{-1} D_{1}\left[\bar{\omega}^{L}(\bar{d})_{M} \mathcal{I}_{N}^{L}+\bar{\omega}^{C}(\bar{d})_{M} \mathcal{I}_{N}^{C}+\bar{\omega}^{R}(\bar{d})_{M} \mathcal{I}_{N}^{R}\right] \mathbf{f}=\mathcal{P}^{-1} \mathcal{Q} \mathbf{f}
$$

The solution to equation (16) is the central objective of this section; specifically, to express a target fourth-order SBP discretization $\mathcal{P}^{-1} \mathcal{Q}$, in terms of the conventional 1) interpolation operators $\left.M_{N} \mathcal{I}_{N}, 2\right)$ weight functions $\bar{\omega}$, and 3) difference operator $D_{1}$ needed to implement the ESWENO formulation.

Remark. There is no guarantee that a solution exists to Equation (16). The conventional periodic domain operators $M \mathcal{I}_{N}^{r}, \bar{\omega}^{r} ; r=L, C, R$ on uniformly spaced grids $\mathbf{x}_{N}, \overline{\mathbf{x}}_{M}$, match the fourth-order centered $\mathcal{P}^{-1} \mathcal{Q}$ identically in the interior of the domain. The task at hand is to determine boundary operators for ${ }_{M} \mathcal{I}_{N}^{r}, \bar{\omega}^{r} ; r=$ $L, C, R$ and the grids $\mathbf{x}_{N}, \overline{\mathbf{x}}_{M}$ that match $\mathcal{P}^{-1} \mathcal{Q}$ at boundaries.

Remark. Equation (16) is a nonlinear function of the flux grid $\overline{\mathbf{x}}_{M}$. Indeed, assuming a uniform solution grid $\mathbf{x}_{N}$, the functional dependency of the matrix operators are

$$
\begin{gathered}
{\left[\overline{\mathbf{d}}^{(r)}=\overline{\mathbf{d}}^{(r)}\left(\overline{\mathbf{x}}_{M}\right) ;{ }_{M} \mathcal{I}_{N}^{(r)}={ }_{M} \mathcal{I}_{N}^{(r)}\left(\overline{\mathbf{x}}_{M}\right)\right], r=L, C, R \quad ;} \\
\mathcal{P}=\mathcal{P}\left(\vartheta_{l}\right), \mathcal{Q}=\mathcal{Q}\left(\vartheta_{l}\right), l=1,2
\end{gathered}
$$

\subsection{Nonuniform Flux-Points}

An analytic solution to equation (16) is not immediately forthcoming using Mathematica 7.0 [17], if the solution $\mathbf{x}_{N}$ and flux $\overline{\mathbf{x}}_{M}$ points are uniformly spaced on the domain $0 \leq x \leq 1$ :

$$
\begin{gathered}
x_{j+1}=j \Delta x, j=0, N-1, \Delta x=1 /(N-1) ; \\
\bar{x}_{k}=\frac{\left(x_{k}+x_{k+1}\right)}{2}, k=1, N-1, \bar{x}_{0}=0, \bar{x}_{N}=1,
\end{gathered}
$$


for either the diagonal-norm or the block-norm SBP operators $\mathcal{P}^{-1} \mathcal{Q}$. Increasing the boundary block sizes to $(6 \times 6)$, while providing numerous additional free parameters $\vartheta_{l}$, does not remove an apparent inconsistency in the constraint equations.

Multiple solutions exist for equation (16), for either the diagonal- or block-norms, if the second, third, and fourth flux points $\overline{\mathbf{x}}_{M}$ are shifted to a nonuniform set of points near the boundaries. (Figure (1) illustrates the relationship between the solution-mesh $\mathbf{x}_{N}$ and flux-mesh $\overline{\mathbf{x}}_{M}$, including the shifted flux points near each boundary.) The location of the flux points is precisely related to the $\mathcal{P}$-norm used to prove stability for the SBP operator. The following lemma is a central contribution of this work:

Lemma 1 Assume $N$ uniformly distributed solution points $\mathbf{x}_{N}$. There exists an $N+$ 1 dimensional set of flux points $\overline{\mathbf{x}}_{M}$ for which the central, fourth-order, ESWENO interpolation operators, can be expressed in SBP form. The discrete set of points $\overline{\mathbf{x}}_{M}$ satisfies the following expression:

$$
\mathcal{P}^{-1} D_{1} \overline{\mathbf{x}}_{M}=\mathbf{1}
$$

with 1 the unit vector.

Proof: The proof is constructive. Given $\overline{\mathbf{x}}_{M}$, the general solutions are determined using Mathematica. Owing to the complexity of the general expressions, only two optimized solutions are included herein: one derived using a diagonal-norm, the other a block-norm. Note that $D_{1} \overline{\mathbf{x}}_{M}=\mathcal{P} \mathbf{1}$ is an equivalent statement because $\mathcal{P}$ is nonsingular.

Although a mathematical motivation for Lemma (1) has not been found, a dimensional argument can be used to explain the relationship between $\mathcal{P}$ and $\overline{\mathbf{x}}_{M}$. First, note that the $D_{1}$ matrix is an undivided difference, and does not contain any reference to the precise location of the flux-points $\overline{\mathbf{f}}$, nor to their separation; $D_{1}$ is dimensionless and simply forms the difference between the left and right cell interface states. The dimensionality (or inverse weight) in the derivative operation is provided by the term $\left[\delta \overline{\mathbf{x}}_{M}\right]^{-1}$. Left multiplying the flux difference $D_{1} \mathbf{f}$ by $\mathbf{1}^{T}\left[\delta \overline{\mathbf{x}}_{M}\right]$ (the matrix equivalent of integration), yields the domain flux balance $\mathbf{1}^{T}\left[\delta \overline{\mathbf{x}}_{M}\right] D_{1} \overline{\mathbf{f}}=f(1)-f(0)$. Clearly, the individual terms in the matrix $\left[\delta \overline{\mathbf{x}}_{M}\right]$ are the quadrature weights needed for discrete integration. Thus, the matrix $\left[\delta \overline{\mathbf{x}}_{M}\right]$ is filling precisely the same role in equation (16), as the $\mathcal{P}$ matrix in the SBP context $D=\mathcal{P}^{-1} \mathcal{Q}$. It is not surprising that $\mathcal{P}$ and $\left[D_{1} \overline{\mathbf{x}}_{M}\right]$ are closely related.

The next two examples demonstrate the validity of Lemma (1) as well as provide the flux points $\overline{\mathbf{x}}_{M}$ needed for the diagonal- and block-norm fourth-order SBP operators. First, consider the $[N \times N]$ diagonal $\mathcal{P}$-norm $\mathcal{P}_{d}$ defined by

$$
\mathcal{P}_{d}=\Delta x \operatorname{Diag}\left[\begin{array}{lllllllllll}
\frac{17}{48} & \frac{59}{48} & \frac{43}{48} & \frac{49}{48} & 1 & \cdots & 1 & \frac{49}{48} & \frac{43}{48} & \frac{59}{48} & \frac{17}{48}
\end{array}\right]
$$

Distribute the flux points $\overline{\mathbf{x}}_{M}$ as

$$
\overline{\mathbf{x}}_{M}=\left[0, \bar{x}_{1}, \bar{x}_{2}, \bar{x}_{3}, \frac{7 \Delta x}{2}, \cdots,\left(1-\frac{7 \Delta x}{2}\right),\left(1-\bar{x}_{3}\right),\left(1-\bar{x}_{2}\right),\left(1-\bar{x}_{1}\right), 1\right]^{T}
$$


where

$\bar{x}_{1}=\bar{x}_{0}+\frac{17 \Delta x}{48} ; \bar{x}_{2}=\bar{x}_{1}+\frac{59 \Delta x}{48} ; \bar{x}_{3}=\bar{x}_{2}+\frac{43 \Delta x}{48} ; \bar{x}_{4}=\bar{x}_{3}+\frac{49 \Delta x}{48}=\frac{7 \Delta x}{2}$.

Then, the vector $D_{1} \overline{\mathbf{x}}_{M}$ is precisely the reciprocal of the diagonal element of $\mathcal{P}_{d}$. The product $\mathcal{P}_{d}^{-1} D_{1} \overline{\mathbf{x}}_{M}$ is trivially the identity vector $\mathbf{1}$, [the condition of Lemma (1)] since $\mathcal{P}_{d}$ is a diagonal matrix.

Lemma (1) generalizes immediately to block-norm $\mathcal{P}$ cases. Recall that $\mathcal{P}$ is not uniquely defined in the fourth-order, centered, block-norm case. The matrix $\mathcal{P}$ is a function of two independent parameters (i.e. $\mathcal{P}=\mathcal{P}\left(\vartheta_{l}\right), l=1,2$ ), after all accuracy, and matrix constraints are satisfied. In this specific case (but not in general) the matrix-vector product $\mathcal{P} \mathbf{1}$, used in Lemma (1) to determine $\overline{\mathbf{x}}_{M}$, is independent of the parameters $\vartheta_{l}$. Thus, the following set of points $\overline{\mathbf{x}}_{M}$

$$
\begin{gathered}
\overline{\mathbf{x}}_{M}=\left[0, \frac{43 \Delta x}{144}, \frac{61 \Delta x}{36}, \frac{349 \Delta x}{144}, \frac{7 \Delta x}{2}, \cdots\right. \\
\left(1-\frac{7 \Delta x}{2}\right),\left(1-\frac{349 \Delta x}{144}\right),\left(1-\frac{61 \Delta x}{36}\right),\left(1-\frac{43 \Delta x}{144}\right), 1^{T}
\end{gathered}
$$

satisfied the conditions of Lemma (1), independent of the values of the available parameters $\vartheta_{l}, l=1,2$.

Remark. Existence of a set of points $\overline{\mathbf{x}}_{M}$ does not imply that $\overline{\mathbf{x}}_{M}$ is unique, although no other set has been identified.

Remark. The solution- $\left(\mathbf{x}_{N}\right)$ and flux- $\left(\overline{\mathbf{x}}_{M}\right)$ grids are interdigitated, the exceptions being the collocated endpoints. Given this set of flux points $\overline{\mathbf{x}}_{M}$, the interpolation stencils $S^{r}, r=\{L, C, R\}$ are consistently defined for all points $\bar{x}_{M_{j}}, j=2, N-2$. The flux-points next to each boundary $\bar{x}_{M_{j}}, j=1$ and $j=N-1$ only have a centered and an inward-biased stencil. The first and last flux-points, in addition to inward biased interpolation stencils, have an "exact" interpolant derived from the collocated solution-point. This property is shared with conventional WENO boundary operators.

\subsection{Interpolation Operators and Preferred Weights}

An analytic solution to equation (16) exists for either the diagonal-norm or the block-norm case, when $\overline{\mathbf{x}}_{M}$ is given by equation (18) or (19), respectively. The diagonal-norm case required additional interpolants at the boundaries, to become realizable. Specifically, the interpolation operators $I^{L L}, I^{R R}$, and weights $\bar{w}^{L L}, \bar{w}^{R R}$, were added in equations (13) and (16). The additional terms were only required at the boundaries.

Interpolation operators for the WENO/ESWENO $3-4-3$ and WENO/ESWENO $2-4-2$ operators are given in the appendix.

\section{Energy Stabilization Terms}

When the nonlinear weights used in equation (13) satisfy the target conditions, $\bar{w}^{(r)}=\bar{\omega}^{(r)}, r=\{L, C, R\}$, then all stencils are stable without the dissipation term $\mathcal{P}^{-1} \mathcal{R}$. With nonlinear weights, however, there is no guarantee that the baseline 
WENO operator $D$ is stable. To ensure stability, an artificial dissipation term $D_{a d}$ is added to the WENO operator $D$, such that the symmetric portion of the resulting (ESWENO) operator $\bar{D}$ is positive-definite. Specifically, $\bar{D}$ satisfies the following matrix conditions: $\bar{D}=D+D_{a d}$ subject to $D_{\text {sym }}+D_{a d} \geq 0$.

The matrix $D_{a d}$ is constructed by expanding the symmetric matrix $D_{\text {sym }}$ into its elemental components using the decomposition ${ }^{4}$

$$
D_{\text {sym }}=\mathcal{P}^{-1}\left[\Lambda_{0}+D_{1} \Lambda_{1}\left[D_{1}\right]^{T}+D_{1}\left[D_{1}\right]^{T} \Lambda_{2} D_{1}\left[D_{1}\right]^{T}+D_{1}\left[D_{1}\right]^{T} D_{1} \Lambda_{3}\left[D_{1}\right]^{T} D_{1}\left[D_{1}\right]^{T}\right] .
$$

The existence of this decomposition is established in Ref. [6].

Next, the diagonal terms of $\Lambda_{i}$ are modified to be smoothly positive using the expression:

$$
\bar{\lambda}_{j}^{(i)}=\frac{1}{2}\left(\sqrt{\left(\lambda_{j}^{(i)}\right)^{2}+\delta_{i}^{2}}-\lambda_{j}^{(i)}\right)
$$

where $\delta$ is dependent on the grid spacing.

$$
\delta_{1}=(\Delta \xi)^{3} \quad \delta_{2}=(\Delta \xi)^{2} \quad \delta_{3}=(\Delta \xi)^{2}
$$

The artificial dissipation operator takes the final form

$$
\begin{gathered}
D_{a d} \mathbf{f}=\mathcal{P}^{-1} \mathcal{R} \mathbf{f}= \\
\mathcal{P}^{-1}\left[\bar{\Lambda}_{0}+D_{1} \bar{\Lambda}_{1}\left[D_{1}\right]^{T}+D_{1}\left[D_{1}\right]^{T} \bar{\Lambda}_{2} D_{1}\left[D_{1}\right]^{T}+D_{1}\left[D_{1}\right]^{T} D_{1} \bar{\Lambda}_{3}\left[D_{1}\right]^{T} D_{1}\left[D_{1}\right]^{T}\right] \mathbf{f}
\end{gathered}
$$

The dissipation matrix $\Lambda_{0}$ (at least herein) is identically zero for both the diagonal and block norm schemes; thus it is convenient to define the vector

$$
\bar{\psi}=\left[\bar{\Lambda}_{1}\left[D_{1}\right]^{T}+\left[D_{1}\right]^{T} \bar{\Lambda}_{2} D_{1}\left[D_{1}\right]^{T}+\left[D_{1}\right]^{T} D_{1} \bar{\Lambda}_{3}\left[D_{1}\right]^{T} D_{1}\left[D_{1}\right]^{T}\right] \mathbf{f}
$$

This definition of $\bar{\psi}$ allows the artificial dissipation term to be combined with the original flux $\overline{\mathbf{f}}$ given by equation (13) to yield the following:

$$
\frac{d \mathbf{f}}{d x}=\mathcal{P}^{-1} D_{1}(\overline{\mathbf{f}}+\bar{\psi})+O\left(\Delta x^{4}\right)
$$

\section{Numerical Tests}

The accuracy and stability characteristics of the new ESWENO framework are tested using the one dimensional advection equation and the quasi-one dimensional and two dimensional Euler equations. As a more practical test, the framework was implemented in a two dimensional reacting Navier-Stokes solver and was used to simulate supersonic non-reacting and reacting shear layers. All simulations are integrated in time or pseudo-time using the same five-step, fourth-order Runge Kutta solver [18].

\footnotetext{
${ }^{4}$ Note that the dimensions of the even indexed $\Lambda_{i}$ are $(N \times N)$, while the odd indexed $\Lambda_{i}$ have dimensions $(N+1 \times N+1)$. This is the result of the difference matrix $D_{1}$ having dimensions $[N \times(N+1)]$.
} 


\subsection{Eigenvalues of the ESWENO operator}

To demonstrate that the finite-domain ESWENO operator is positive semidefinite, an eigenvalue analysis is conducted using the one dimensional scalar advection equation with discontinuous initial data. We begin by rearranging equation (2) into the form

$$
\mathcal{P} \frac{d \overline{\mathbf{u}}}{d t}=-a(\mathcal{Q}+\mathcal{R}) \overline{\mathbf{u}}
$$

Setting $\overline{\mathbf{u}}=\hat{\mathbf{u}} e^{k t}$ (see Ref. [19]) in equation (25) yields

$$
-\lambda \mathcal{P} \hat{\mathbf{u}}=(\mathcal{Q}+\mathcal{R}) \hat{\mathbf{u}}, \quad \lambda=\frac{k}{a}
$$

Stability is guaranteed if all real eigenvalues satisfy the condition $\lambda_{r} \leq 0$ since $a>0$. ESWENO/WENO operators depend on the calculated solution, so eigenvalues were calculated over ten characteristic times using $N=100$ grid cells to ensure consistency of the analysis. The boundary condition used in the simulation was $g(t)=0$. Eigenvalues were computed for the ESWENO/WENO $3-4-3$ scheme applied to a convected square wave. The resulting maximum real eigenvalues are plotted against time in Figure (2).

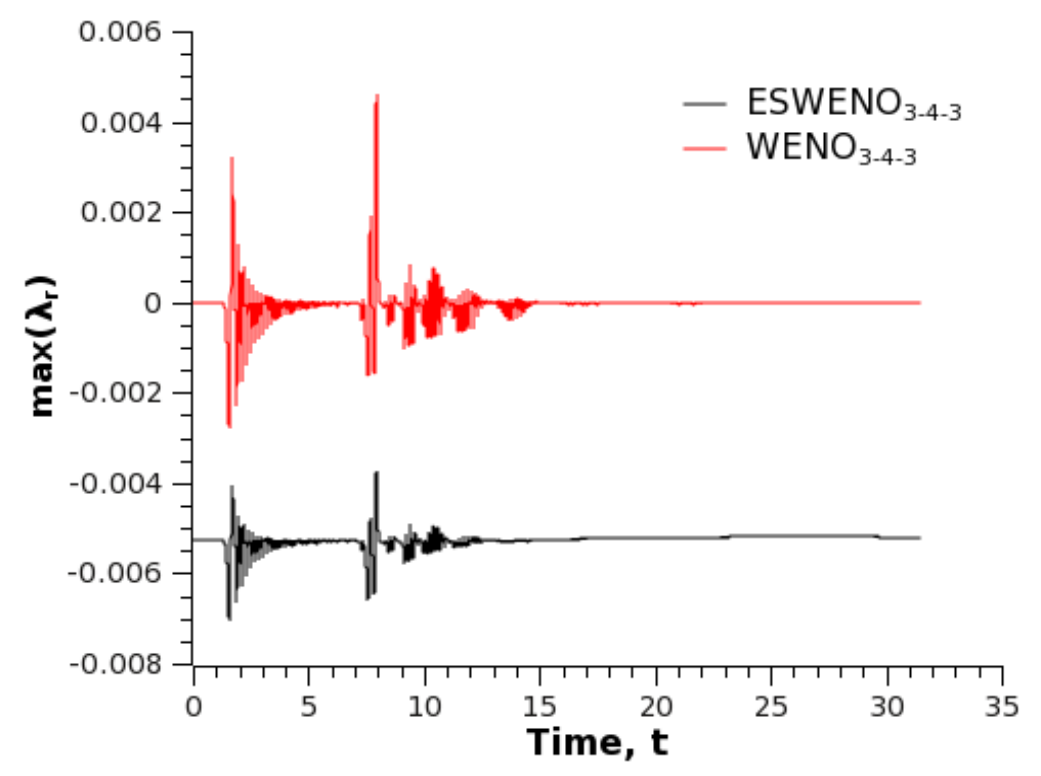

Figure 2. Maximum real eigenvalues for a convected square wave are plotted as a function of time for the $\mathrm{WENO}_{3-4-3}$ operator with and without the energy stable addition.

It is clear that the bounded $\mathrm{WENO}_{3-4-3}$ operator has eigenvalues in the right half of the complex plane. This is fixed by the addition of the energy stable term in the ESWENO $3-4-3$ scheme. This result is similar to that observed in the periodic case $[5,6]$. 


\subsection{Linear Wave Equation}

Numerical solutions of the linear advection equation were examined to test the accuracy and dissipation characteristics of the finite-domain ESWENO schemes. The convergence rate of the $\mathrm{ESWENO}_{2-4-2}$ and $\mathrm{ESWENO}_{3-4-3}$ operators are calculated for a smooth solution. To evaluate the robustness of the schemes, a wave solution with a time-dependent, discontinuous inlet condition is examined. It is important to note that since the ESWENO $3-4-3$ operator is constructed using a block P-norm, simply assigning the boundary solution to be the boundary data (injection boundary conditions) does not maintain energy stability. Instead, both ESWENO schemes utilize the Simultaneous Approximation Term (SAT) penalty method to enforce the boundary conditions [20].

\subsubsection{Sine Wave}

To test the global order of convergence, the ESWENO scheme is used to approximate the solution to the linear advection equation with the exact solution

$$
u(x, t)=\sin (x-a t), \quad a=1, \quad x \in(-\pi, \pi)
$$

Ten characteristic times were simulated. The $L_{2}$ and $L_{\infty}$ errors are tabulated along with the convergence rate in Tables (1) and (2), respectively, for the full block-norm ESWENO operator and are compared to the errors from the block-norm linear centered difference operator. For the diagonal norm operators, the errors are tabulated in Tables (3) and (4). It is clear that the ESWENO operator yields the design order of convergence for the linear advection equation, and provides almost the same accuracy as the linear counterpart.

Table 1. $L_{2}$ error and convergence rate of a smooth solution to the $1 \mathrm{D}$ advection equation for the linear and ESWENO operators with the block norm boundary closure.

\begin{tabular}{ccccc}
\hline \multirow{2}{*}{ Number of Cells } & \multicolumn{2}{c}{ Linear Block Norm } & \multicolumn{2}{c}{ ESWENO $_{3-4-3}$} \\
\cline { 2 - 5 } & $\mathbf{L}_{\mathbf{2}}$ Error & $\mathbf{L}_{\mathbf{2}}$ Rate & $\mathbf{L}_{\mathbf{2}}$ Error & $\mathbf{L}_{\mathbf{2}}$ Rate \\
\hline \hline 100 & $1.60 \mathrm{e}-06$ & - & $1.65 \mathrm{e}-06$ & - \\
\hline 200 & $1.01 \mathrm{e}-07$ & 3.99 & $1.04 \mathrm{e}-07$ & 3.99 \\
\hline 400 & $6.31 \mathrm{e}-09$ & 4.00 & $6.55 \mathrm{e}-09$ & 3.99 \\
\hline 800 & $3.95 \mathrm{e}-10$ & 4.00 & $4.10 \mathrm{e}-10$ & 4.00 \\
\hline 1600 & $2.52 \mathrm{e}-11$ & 3.97 & $2.61 \mathrm{e}-11$ & 3.97 \\
\hline
\end{tabular}

\subsubsection{Square Wave}

A square wave advection problem with the exact solution

$$
u(x, t)=\frac{\tanh (b(x-a t+2 \pi))-\tanh \left(b\left(x-a t+\frac{3 \pi}{2}\right)\right)}{2}, \quad a=1, \quad x \in(-\pi, \pi)
$$


Table 2. $L_{\infty}$ error and convergence rate of a smooth solution to the $1 \mathrm{D}$ advection equation for the linear and ESWENO operators with the block norm boundary closure.

\begin{tabular}{ccccc}
\hline \multirow{2}{*}{ Number of Cells } & \multicolumn{2}{c}{ Linear Block Norm } & \multicolumn{2}{c}{ ESWENO } \\
\cline { 2 - 5 } & $\mathbf{L}_{\infty}$ Error & $\mathbf{L}_{\infty}$ Rate & $\mathbf{L}_{\infty}$ Error & $\mathbf{L}_{\infty}$ Rate \\
\hline \hline 100 & $3.24 \mathrm{e}-06$ & - & $3.43 \mathrm{e}-06$ & - \\
\hline 200 & $2.07 \mathrm{e}-07$ & 3.97 & $2.18 \mathrm{e}-07$ & 3.97 \\
\hline 400 & $1.30 \mathrm{e}-08$ & 3.99 & $1.38 \mathrm{e}-08$ & 3.99 \\
\hline 800 & $8.17 \mathrm{e}-10$ & 3.99 & $8.65 \mathrm{e}-10$ & 3.99 \\
\hline 1600 & $5.20 \mathrm{e}-11$ & 3.97 & $5.51 \mathrm{e}-11$ & 3.97 \\
\hline
\end{tabular}

Table 3. $L_{2}$ error and convergence rate of a smooth solution to the $1 \mathrm{D}$ advection equation for the linear and ESWENO operators with the block norm boundary closure.

\begin{tabular}{ccccc}
\hline \multirow{2}{*}{ Number of Cells } & \multicolumn{2}{c}{ Linear Diagonal Norm } & \multicolumn{2}{c}{ ESWENO } \\
\cline { 2 - 5 } & $\mathbf{L}_{\mathbf{2}}$ Error & $\mathbf{L}_{\mathbf{2}}$ Rate & $\mathbf{L}_{\mathbf{2}}$ Error & $\mathbf{L}_{\mathbf{2}}$ Rate \\
\hline \hline 100 & $4.20 \mathrm{e}-05$ & - & $2.05 \mathrm{e}-05$ & - \\
\hline 200 & $5.21 \mathrm{e}-06$ & 3.01 & $2.30 \mathrm{e}-06$ & 3.16 \\
\hline 400 & $6.48 \mathrm{e}-07$ & 3.01 & $2.72 \mathrm{e}-07$ & 3.08 \\
\hline 800 & $8.09 \mathrm{e}-08$ & 3.00 & $3.30 \mathrm{e}-08$ & 3.04 \\
\hline 1600 & $1.01 \mathrm{e}-08$ & 3.00 & $4.07 \mathrm{e}-09$ & 3.02 \\
\hline
\end{tabular}

was tested, where $b=200$ caused a very sharp gradient that could not be resolved. The numerical solution was calculated for up to $t=10.0$, and $N=200$ grid cells were used. The initial condition was $u_{0}(x, 0)=0$ in the domain. The wave was passed into the domain, advected through the interior, and then passed out of the domain. This problem tests the dissipation and oscillatory characteristics of the boundary treatment as well as the interior treatment. A comparison of the numerical solution to the exact solution is shown for the two different boundary norms in Figure (3) at two different times. The solutions shown in Figure (3) prevent oscillations, and smearing of the discontinuity caused by the boundary treatment, particularly at the inlet, is reasonable. The solution using the $\mathrm{ESWENO}_{2-4-2}$ operator in the left figure $(t=2.0)$ exhibits slightly more dissipative error than the solution using the $\mathrm{ESWENO}_{3-4-3}$ operator.

\subsection{Euler Equations}

The quasi-one dimensional Euler equations given by

$$
\begin{gathered}
\frac{\partial \mathbf{U}}{\partial t}+\frac{\partial \mathbf{F}}{\partial x}=\frac{A_{x}}{A} \mathbf{G} \\
\mathbf{U}=\left(\begin{array}{c}
\rho \\
\rho u \\
\rho E
\end{array}\right) \quad \mathbf{F}=\left(\begin{array}{c}
\rho u \\
\rho u u+p \\
(\rho E+p) u
\end{array}\right) \mathbf{G}=\left(\begin{array}{c}
\rho u \\
\rho u u \\
(\rho E+p) u
\end{array}\right)
\end{gathered}
$$


Table $4 . L_{\infty}$ error and convergence rate of a smooth solution to the $1 \mathrm{D}$ advection equation for the linear and ESWENO operators with the block norm boundary closure.

\begin{tabular}{ccccc}
\hline \multirow{2}{*}{ Number of Cells } & \multicolumn{2}{c}{ Linear Diagonal Norm } & \multicolumn{2}{c}{ ESWENO $_{2-4-2}$} \\
\cline { 2 - 5 } & $\mathbf{L}_{\infty}$ Error & $\mathbf{L}_{\infty}$ Rate & $\mathbf{L}_{\infty}$ Error & $\mathbf{L}_{\infty}$ Rate \\
\hline \hline 100 & $7.79 \mathrm{e}-05$ & - & $6.60 \mathrm{e}-05$ & - \\
\hline 200 & $9.96 \mathrm{e}-06$ & 2.97 & $8.16 \mathrm{e}-06$ & 3.02 \\
\hline 400 & $1.25 \mathrm{e}-06$ & 3.00 & $1.07 \mathrm{e}-06$ & 2.93 \\
\hline 800 & $1.57 \mathrm{e}-07$ & 3.00 & $1.37 \mathrm{e}-07$ & 2.97 \\
\hline 1600 & $1.96 \mathrm{e}-08$ & 3.00 & $1.73 \mathrm{e}-08$ & 2.99 \\
\hline
\end{tabular}

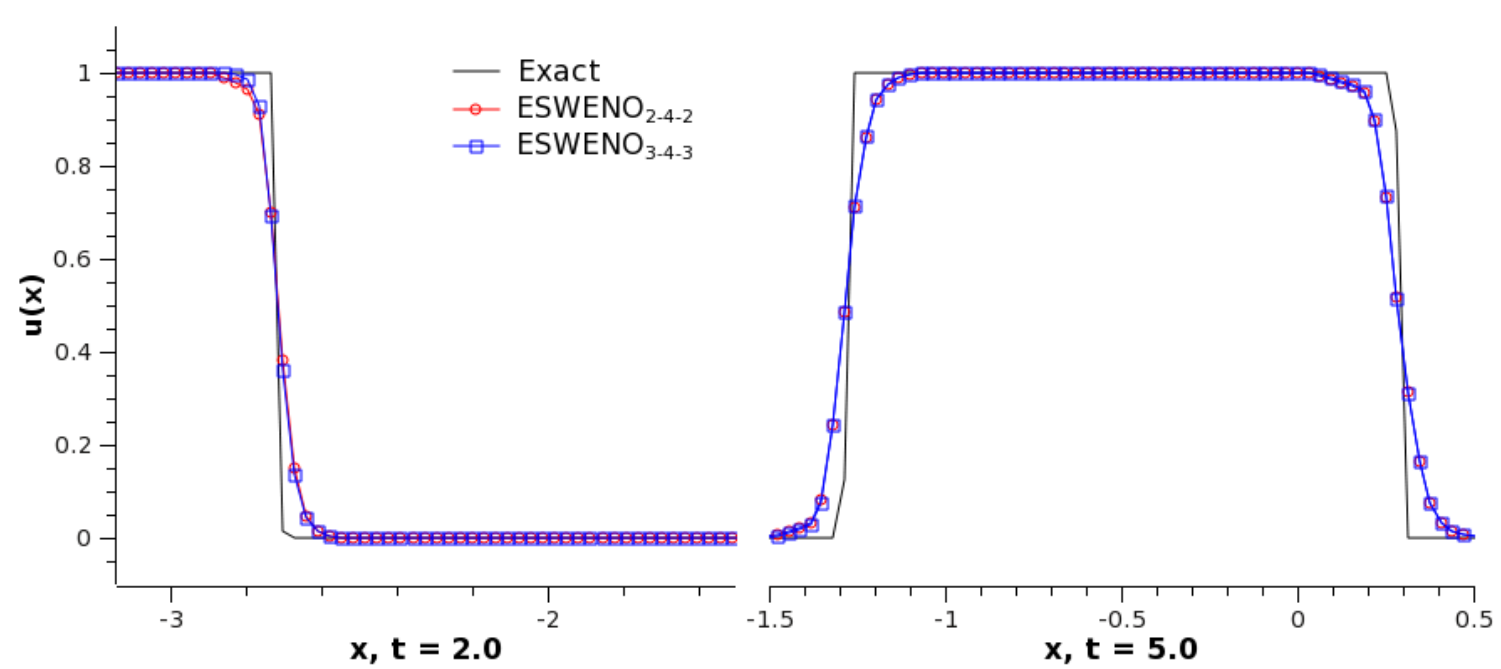

Figure 3. Numerical solutions using the $\mathrm{ESWENO}_{2-4-2}$ and $\mathrm{ESWENO}_{3-4-3}$ operator compared to the exact solution of an advected square wave at two different times.

were used to test the extension of the ESWENO operators to non-linear hyperbolic systems of equations. In order to properly calculate stencil biasing parameters, the conservative fluxes in equation (29) are transformed into characteristic form and split into forward and backward propagating waves using global Lax-Friedrichs splitting.

$$
\mathbf{f}^{ \pm}=\frac{1}{2}\left(\mathbf{F}_{\mathbf{c}} \pm \Lambda_{\max } \mathbf{U}_{\mathbf{c}}\right)
$$

where $\mathbf{F}_{\mathbf{c}}$ and $\mathbf{U}_{\mathbf{c}}$ represent the characteristic fluxes and variables, respectively. The transformation from conservative to characteristic variables is performed at each flux point using transformation matrices constructed from the Roe averaged variables at the flux point. The ESWENO reconstruction is used to calculate interpolated fluxes, and then the interpolated fluxes are transformed back to physical space, where the gradient is calculated.

The solutions to the quasi-1D Euler equations utilize the SAT penalty boundary 
conditions in order to ensure stability of the solution and consistency of the boundary closure. The details of the SAT penalty method for the Euler and Navier-Stokes equations can be found in Svärd, Carpenter, and Nordström [21].

\subsubsection{Subsonic One-Dimensional Nozzle}

The first nonlinear test problem was a steady state, subsonic flow in a one-dimensional nozzle. The area was defined as

$$
A(x)=1-0.8 x(1-x), \quad x \in(0,1)
$$

The inlet Mach number was set to $M=0.5$ and the outlet pressure was set to be equivalent to the inlet. The exact solution is plotted in Figure (4). The $L_{2}$ error norm of the density and the convergence rate for both ESWENO schemes is tabulated in Table (5). The calculated convergence rates match the design order.

Remark. The design order of convergence is achieved despite the fact that the transformation matrices are calculated from second order accurate Roe averages. This is because the transformation is only used to transform to wave space and back to physical space in order to facilitate flux splitting. The accuracy of this transformation does not affect the order of accuracy of the interpolation. Instead, the accuracy of the transformation relates to how accurately the physical fluxes can be decomposed into waves.

Table 5. $L_{2}$ error and convergence rate of a smooth solution to the quasi-one dimensional Euler equations for $\mathrm{ESWENO}_{2-4-2}$ and $\mathrm{ESWENO}_{3-4-3}$.

\begin{tabular}{ccccc}
\hline \multirow{2}{*}{ Number of Cells } & \multicolumn{2}{c}{ ESWENO $_{2-4-2}$} & \multicolumn{2}{c}{ ESWENO $_{3-4-3}$} \\
\cline { 2 - 5 } & $\mathbf{L}_{\mathbf{2}}$ Error & $\mathbf{L}_{\mathbf{2}}$ Rate & $\mathbf{L}_{\mathbf{2}}$ Error & $\mathbf{L}_{\mathbf{2}}$ Rate \\
\hline \hline 25 & $6.55 \mathrm{E}-05$ & - & $2.13 \mathrm{E}-06$ & - \\
\hline 50 & $6.14 \mathrm{E}-06$ & 3.42 & $1.14 \mathrm{E}-07$ & 4.22 \\
\hline 100 & $8.05 \mathrm{E}-07$ & 2.93 & $6.43 \mathrm{E}-09$ & 4.15 \\
\hline 200 & $1.03 \mathrm{E}-07$ & 2.97 & $3.82 \mathrm{E}-10$ & 4.07 \\
\hline
\end{tabular}

\subsubsection{Transonic One-Dimensional Nozzle}

The next test of the boundary-closed ESWENO scheme was a steady state transonic one-dimensional nozzle. The cross sectional area of the transonic nozzle is

$$
A(x)=1.398+0.347 \tanh (0.8 x-4), \quad x \in(0,10)
$$

The inlet Mach number was set to $M=1.5$, and the shock through the overexpanded nozzle occurred at $x=5.0$. The resulting density profile from each ESWENO scheme is compared to the exact solution in Figure (5). The solution is non-oscillatory, and spreads the shock over a few cells. The results from the different boundary treatments do not differ appreciably, which can be attributed to the zero gradient at the boundary. 


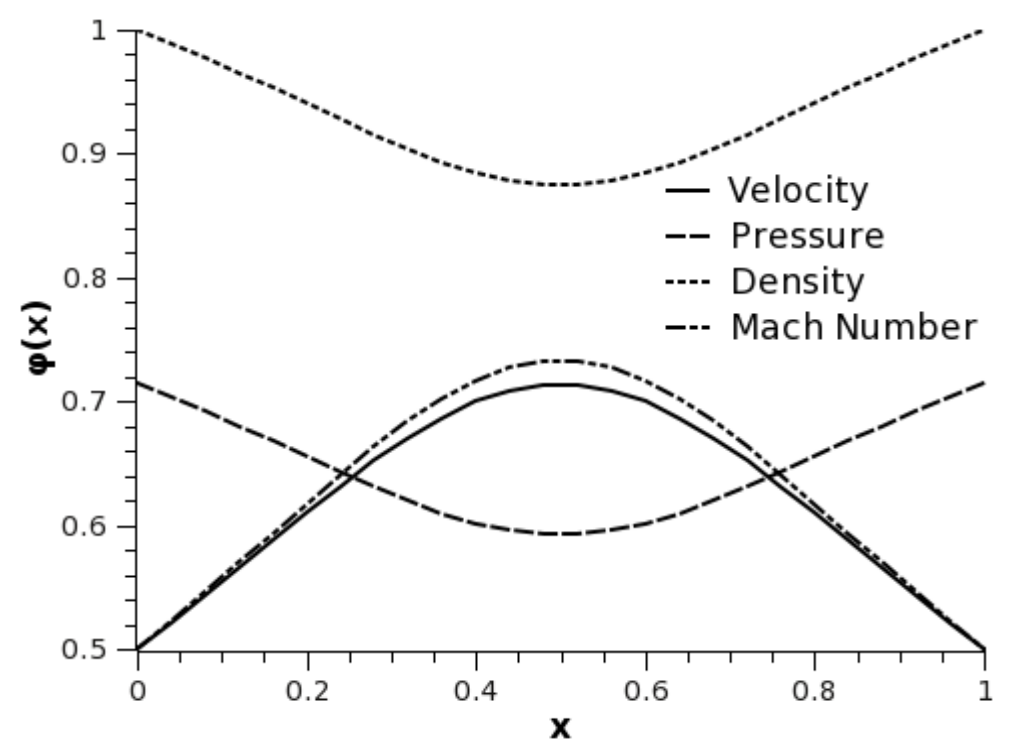

Figure 4. Solution for the quasi-1D subsonic nozzle problem is shown.

Remark. The transonic one-dimensional nozzle is a problem where boundary effects do not significantly influence the solution, but the problem demonstrates the stability of the ESWENO boundary closure for a steady state problem. The behavior of the solution to this problem in the current work exhibits the same characteristics that were observed in the periodic case [5]. Similarly, Sod's shock tube problem and the sine-shock wave interaction problems exhibited the same characteristics. The results for those problems are not repeated here.

\subsubsection{Two Dimensional Inviscid Vortex}

An inviscid two-dimensional vortex convection problem was used to test the accuracy of the finite-domain ESWENO framework in multiple dimensions. The exact solution to the convected vortex is

$$
\begin{gathered}
f(x, y, t)=1-\left(\left(x-x_{0}-U_{\infty} t\right)^{2}+\left(y-y_{0}\right)^{2}\right) \\
\rho(x, y, t)=\left(1-\epsilon^{2} \frac{\gamma-1}{8 \pi^{2}} \exp (f(x, y, t))\right)^{\frac{1}{\gamma-1}}, \quad p=\frac{\rho^{\gamma}}{\gamma} \\
u(x, y, t)=U_{\infty}-\epsilon \frac{y-y_{0}}{2 \pi} \exp \left(\frac{f(x, y, t)}{2}\right), \\
v(x, y, t)=\epsilon \frac{x-x_{0}-U_{\infty} t}{2 \pi} \exp \left(\frac{f(x, y, t)}{2}\right), \\
U_{\infty}=M_{\infty} c_{\infty}, \quad x \in(0,10), \quad y \in(-5,5), \quad\left(x_{0}, y_{0}\right)=(5,0), \quad t \geq 0
\end{gathered}
$$

where $\epsilon=1.0, M_{\infty}=0.5$, and $\gamma=1.4$ were used. Density contours from the initial condition and the approximate solution at $t=10$ for the $\mathrm{ESWENO}_{3-4-3}$ operator with $N=200 \times 200$ grid cells are shown in Figure (6). 


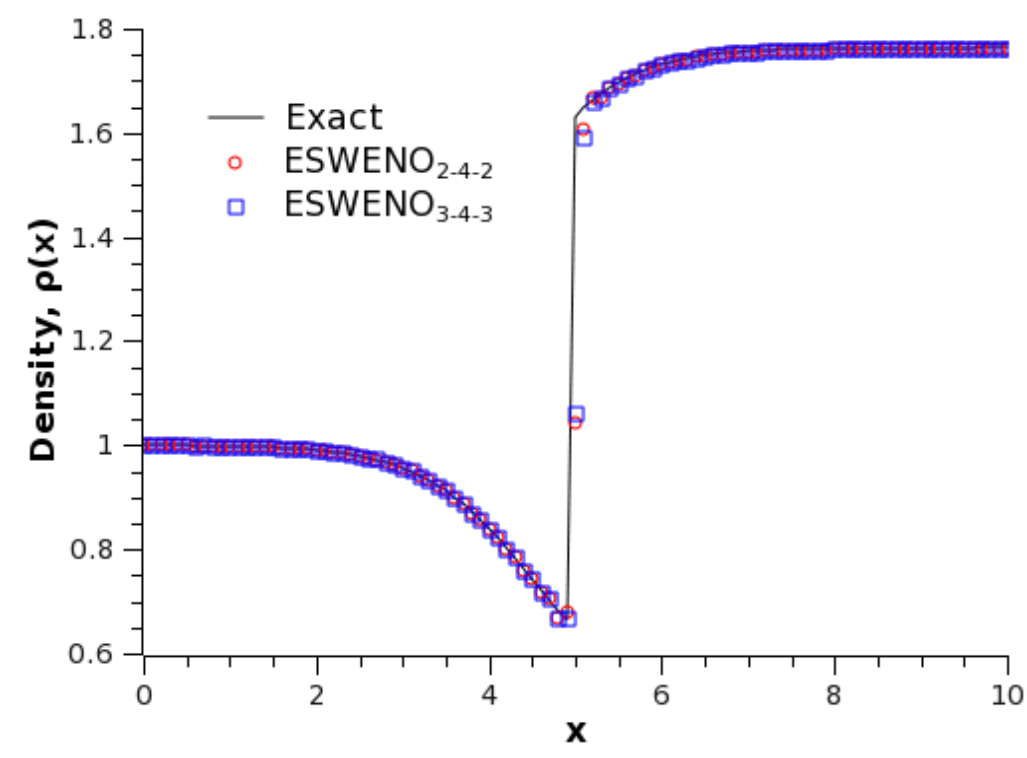

Figure 5. Density is plotted for Sod's shock tube problem at $t=0.2$ using $N=100$ cells.

Both boundary closures were tested for the two-dimensional vortex and compared to corresponding linear operators. The accuracy is affected not only by the boundary closure of the finite difference scheme, but also by the treatment of the boundary conditions. In this case, the SAT penalty method is used to specify the exact boundary data in a well posed manner. This is meant to limit reflections from the boundaries to be influenced only by the boundary closure and the SAT penalty term. A convergence study would not yield meaningful results using more common boundary condition treatments, such as Navier-Stokes Characteristic Boundary Conditions.

The $L_{2}$ and $L_{\infty}$ convergence rates for the $\mathrm{ESWENO}_{3-4-3}$ operator are tabulated in Tables (6) and (7), respectively. The convergence rates for the ESWENO $2-4-2$ operator are given in Tables (8) and (9). The results show that the linear operators recover the design order accuracy. The ESWENO operators yield better than design order convergence rates for lower resolutions, but achieve design order convergence as resolution increases. The ESWENO operators exhibit larger errors for lower resolution than the corresponding linear operator. However, as the critical regions are better resolved, the linear operators and ESWENO operators yield similar $L_{\infty}$ errors and nearly identical $L_{2}$ errors. This is the expected behavior, since the nonlinear weights in the ESWENO operator approach the target weights as resolution is improved, recovering the linear operator.

\subsection{Supersonic Shear Layers}

A more practical test of ESWENO is the two dimensional non-reacting and reacting supersonic hydrogen-air shear layers. These cases test the behavior of the new 


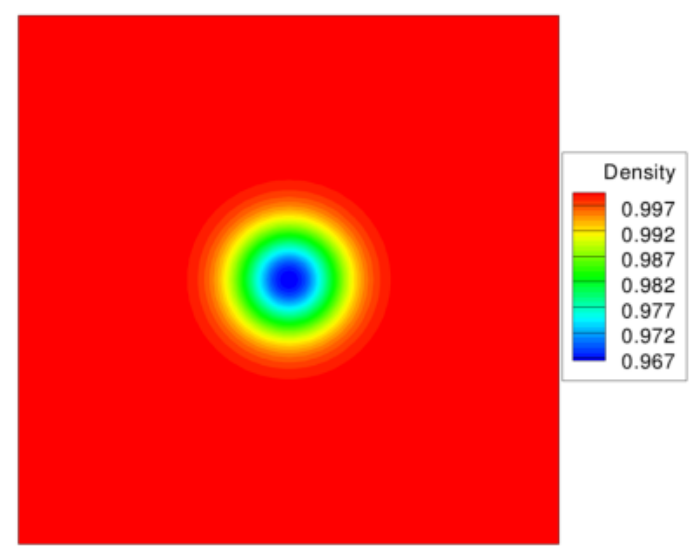

(a) $t=0$

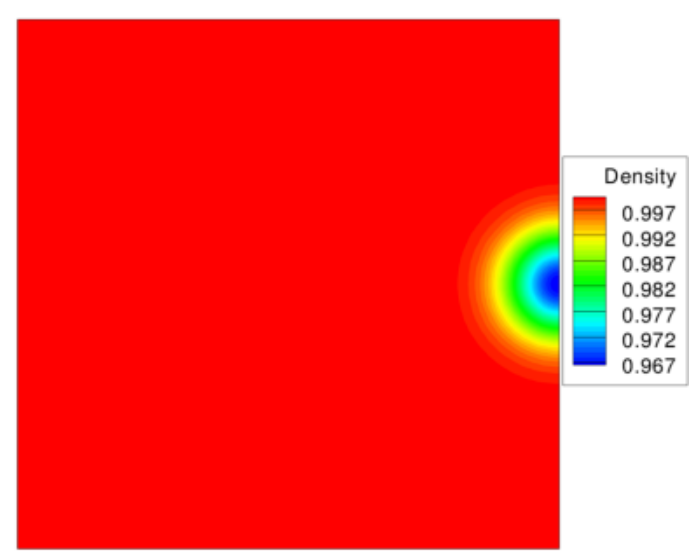

(b) $t=10$

Figure 6. Density contours are shown for a two dimensional isentropic vortex solved using ESWENO $3-4-3$

Table 6. $L_{2}$ error and convergence rates for the $\mathrm{ESWENO}_{3-4-3}$ and linear block norm centered difference operators for the two dimensional inviscid vortex.

\begin{tabular}{ccccc}
\hline \multirow{2}{*}{ Number of Cells } & \multicolumn{2}{c}{ Linear Block Norm } & \multicolumn{2}{c}{ ESWENO $_{3-4-3}$} \\
\cline { 2 - 5 } & $\mathbf{L}_{\mathbf{2}}$ Error & $\mathbf{L}_{\mathbf{2}}$ Rate & $\mathbf{L}_{\mathbf{2}}$ Error & $\mathbf{L}_{\mathbf{2}}$ Rate \\
\hline \hline $50 \times 50$ & $2.49 \mathrm{E}-05$ & - & $5.32 \mathrm{E}-05$ & - \\
\hline $100 \times 100$ & $1.64 \mathrm{E}-06$ & 3.92 & $2.25 \mathrm{E}-06$ & 4.57 \\
\hline $200 \times 200$ & $1.04 \mathrm{E}-07$ & 3.98 & $1.08 \mathrm{E}-07$ & 4.37 \\
\hline $400 \times 400$ & $6.57 \mathrm{E}-09$ & 3.99 & $6.62 \mathrm{E}-09$ & 4.04 \\
\hline
\end{tabular}

scheme where shocks, high gradients, and physical instabilities are present and interact. A successful scheme must provide enough dissipation to damp oscillations near shocks and unresolved species and temperature gradients, but not be so dissipative that it damps the physical Kelvin-Helmholtz instability, which mixes the fuel and oxidizer streams. In addition to shocks and high gradients, the cases presented here include a discontinuous inlet condition and cannot be solved using standard centered finite differences without the addition of filtering and limiters.

The hydrogen air mixing layer used for this study does not include any forcing of the inlet conditions. The top stream has molar species fractions $X_{H_{2}}=0.5$ and $X_{N 2}=0.5$, with a temperature of $T=300 \mathrm{~K}$. The bottom stream is composed of pure air, with inlet temperature of $T=300 \mathrm{~K}$ for non-reacting flow and $T=1500 \mathrm{~K}$ for reacting flow. The Mach number of the top stream is $M=2.0$, while the bottom stream is $M=1.2$. The full two dimensional compressible Navier-Stokes equations were solved. ESWENO was used to calculate convective gradients, while a 4th order, wide stencil SBP centered difference was used to calculate gradients of the viscous terms. The species were assumed to be thermally perfect ideal gases, and the open-source thermodynamics library Cantera [22] was used to calculate thermodynamic and transport properties. A hydrogen air chemical kinetic mechanism 
Table 7. $L_{\infty}$ error and convergence rates for the $\mathrm{ESWENO}_{3-4-3}$ and linear block norm centered difference operators for the two dimensional inviscid vortex.

\begin{tabular}{ccccc}
\hline \multirow{2}{*}{ Number of Cells } & \multicolumn{2}{c}{ Linear Block Norm } & \multicolumn{2}{c}{ ESWENO $_{3-4-3}$} \\
\cline { 2 - 5 } & $\mathbf{L}_{\infty}$ Error & $\mathbf{L}_{\infty}$ Rate & $\mathbf{L}_{\infty}$ Error & $\mathbf{L}_{\infty}$ Rate \\
\hline \hline $50 \times 50$ & $2.46 \mathrm{E}-04$ & - & $4.91 \mathrm{E}-04$ & - \\
\hline $100 \times 100$ & $1.68 \mathrm{E}-05$ & 3.87 & $3.31 \mathrm{E}-05$ & 3.89 \\
\hline $200 \times 200$ & $1.07 \mathrm{E}-06$ & 3.97 & $1.53 \mathrm{E}-06$ & 4.44 \\
\hline $400 \times 400$ & $6.71 \mathrm{E}-08$ & 4.00 & $9.04 \mathrm{E}-08$ & 4.08
\end{tabular}

Table 8. $L_{2}$ error and convergence rates for the $\mathrm{ESWENO}_{2-4-2}$ and linear diagonal norm centered difference operators for the two dimensional inviscid vortex.

\begin{tabular}{ccccc}
\hline \multirow{2}{*}{ Number of Cells } & \multicolumn{2}{c}{ Linear Diagonal Norm } & \multicolumn{2}{c}{ ESWENO $2-4-2$} \\
\cline { 2 - 5 } & $\mathbf{L}_{\mathbf{2}}$ Error & $\mathbf{L}_{\mathbf{2}}$ Rate & $\mathbf{L}_{\mathbf{2}}$ Error & $\mathbf{L}_{\mathbf{2}}$ Rate \\
\hline \hline $50 \times 50$ & $3.49 \mathrm{E}-05$ & - & $6.48 \mathrm{E}-05$ & - \\
\hline $100 \times 100$ & $3.05 \mathrm{E}-06$ & 3.52 & $4.43 \mathrm{E}-06$ & 3.87 \\
\hline $200 \times 200$ & $3.40 \mathrm{E}-07$ & 3.17 & $3.64 \mathrm{E}-07$ & 3.60 \\
\hline $400 \times 400$ & $4.13 \mathrm{E}-08$ & 3.04 & $4.16 \mathrm{E}-08$ & 3.13 \\
\hline
\end{tabular}

with 13 chemical species and 25 reactions was used.

The density contours for the supersonic shear layers are shown in Figure (7). In both cases, the Kelvin-Helmholtz instability is clearly predicted, and the solution is stable despite the presence of shocks and unresolved species and temperature gradients. This suggests that the new ESWENO scheme exhibits the robustness required for chemically reacting calculations without adding excessive numerical dissipation.

\section{Conclusions}

A general strategy is presented in Refs. [5] and [6], for constructing Energy Stable Weighted Essentially Non-Oscillatory (ESWENO) finite difference schemes on periodic domains. ESWENO schemes up to eighth-order are developed and proved to be stable in the energy norm for both continuous and discontinuous solutions of systems of linear hyperbolic equations. Herein, boundary closures are developed for the fourth-order ESWENO scheme that maintain wherever possible the WENO stencil biasing properties, while satisfying the summation-by-parts (SBP) operator convention, thereby ensuring stability in an $L_{2}$ norm. A novel set of non-uniform flux interpolation points is necessary near the boundaries to simultaneously achieve 1) accuracy, 2) the SBP convention, and 3) WENO stencil biasing mechanics. The novelty lies in the recognition that the discrete set of flux points $\overline{\mathbf{x}}_{M}$ must be consistent with the stability norm $\mathcal{P}$ used in the SBP formulation, and in fact are derived from the norm itself. Using the new flux-points $\overline{\mathbf{x}}_{M}$, second-order, and third-order boundary closures are developed that achieve stability in a diagonal- and block-norm sense, respectively. 
Table $9 . L_{\infty}$ error and convergence rates for the $\mathrm{ESWENO}_{2-4-2}$ and linear diagonal norm centered difference operators for the two dimensional inviscid vortex.

\begin{tabular}{ccccc}
\hline \multirow{2}{*}{ Number of Cells } & \multicolumn{2}{c}{ Linear Diagonal Norm } & \multicolumn{2}{c}{ ESWENO $_{2-4-2}$} \\
\cline { 2 - 5 } & $\mathbf{L}_{\infty}$ Error & $\mathbf{L}_{\infty}$ Rate & $\mathbf{L}_{\infty}$ Error & $\mathbf{L}_{\infty}$ Rate \\
\hline \hline $50 \times 50$ & $3.17 \mathrm{E}-04$ & - & $5.48 \mathrm{E}-04$ & - \\
\hline $100 \times 100$ & $2.42 \mathrm{E}-05$ & 3.71 & $4.46 \mathrm{E}-05$ & 3.62 \\
\hline $200 \times 200$ & $2.28 \mathrm{E}-06$ & 3.41 & $3.11 \mathrm{E}-06$ & 3.84 \\
\hline $400 \times 400$ & $2.42 \mathrm{E}-07$ & 3.23 & $2.74 \mathrm{E}-07$ & 3.51 \\
\hline
\end{tabular}

Extensive numerical validation is presented to assess the efficacy of the new boundary closures; the test problems included 1) unsteady 1-D linear wave equation (hyperbolic), 2) steady quasi one-dimensional nozzle flow solving the nonlinear Euler equations, 3) unsteady propagation of a 2-D Euler vortex, and 4) unsteady convection-diffusion-reaction of a supersonic hydrogen-air mixing layer. The global accuracy of the second-order diagonal-norm operators and third-order closures block-norm operators, is shown to be "design-order"; i.e. three and four, respectively, for all test cases. Furthermore, it is shown that the accuracy of the new ESWENO operators is very close to that of the linear target operator. Finally, an eigenvalue solver is used to show that the ESWENO discrete operator is stable for smooth and discontinuous initial data.

Four appendices are included to provide detailed instructions on the implementation of the new ESWENO scheme. The appendices include sections describing the 1) smoothness indicators used by ESWENO, including near-wall stencil definitions, 2) a numerical recipe section that includes pseudo-code for the implementation of ESWENO on nonlinear hyperbolic wave equations, (i.e. Euler), 3) the diagonalnorm interpolation and weights formulae, and the dissipation coefficients needed to stabilize the baseline WENO approach, and 4) the block-norm interpolation and weights formulae, and the dissipation coefficients needed to stabilize the baseline WENO approach. 
(a) Non-Reacting Flow

Density: $\begin{array}{lllllllll}0.16 & 0.22 & 0.28 & 0.34 & 0.4 & 0.46 & 0.52 & 0.58 & 0.64\end{array}$

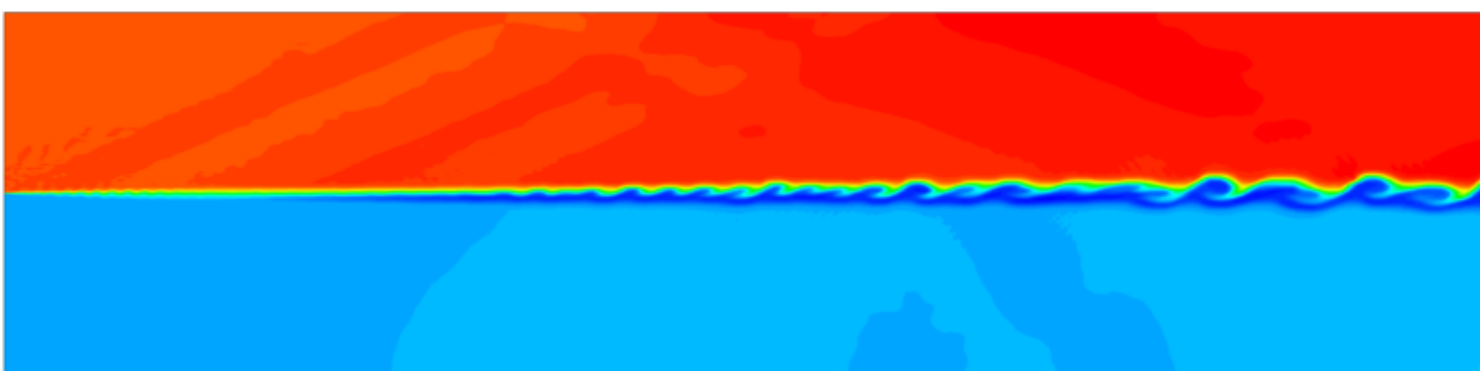

(b) Reacting Flow

Figure 7. Density contours for a non-reacting and reacting supersonic shear layer utilizing fourth order ESWENO.

\section{Appendix A}

\section{Smoothness Indicators}

The smoothness indicators, $\bar{\beta}_{j+1 / 2}^{(r)}$, are calculated at all flux points using the expressions

$$
\begin{gathered}
\bar{\beta}_{j+1 / 2}^{L}=\left(f_{j}-f_{j-1}\right)^{2} \quad \bar{\beta}_{j+1 / 2}^{C}=\left(f_{j+1}-f_{j}\right)^{2} \quad \bar{\beta}_{j+1 / 2}^{R}=\left(f_{j+2}-f_{j+1}\right)^{2} \\
\bar{\beta}_{j+1 / 2}^{L L}=\left(f_{j-1}-f_{j-2}\right)^{2} \quad \bar{\beta}_{j+1 / 2}^{R R}=\left(f_{j+3}-f_{j+2}\right)^{2}
\end{gathered}
$$

(Note that the indicators $\bar{\beta}^{L L}$ and $\bar{\beta}^{R R}$ are only used near the boundaries, and then only when a diagonal matrix norm is being used to prove stability.)

To guarantee that the downwind stencil weight does not exceed that of the central or upwind weights, the downwind smoothness indicator is modified using 
the expression

$$
\bar{\beta}_{j+1 / 2}^{d}=\left(\frac{1}{3} \sum_{r}\left[\bar{\beta}_{j+1 / 2}^{(r)}\right]^{k}\right)^{1 / k},
$$

where $k$ is an even integer.

An additional stencil biasing parameter, $\bar{\tau}_{j+1 / 2}$, is needed for the ESWENO scheme. Here, $\bar{\tau}_{j+1 / 2}$ is a quadratic function of the highest undivided difference available on the stencil for $\bar{f}_{j+1 / 2}$. From flux points $x_{f_{2}}$ to $x_{f_{N-2}}$ [see Fig. (1)],

$$
\bar{\tau}_{j+1 / 2}=\left(-f_{j-1}+3 f_{j}-3 f_{j+1}+f_{j+2}\right)^{2}
$$

At the point $x_{f_{1}}, \bar{\tau}_{j+1 / 2}$ is biased toward the interior of the domain,

$$
\bar{\tau}_{j+1 / 2}=\left(-f_{j}+3 f_{j+1}-3 f_{j+2}+f_{j+3}\right)^{2},
$$

and at $x_{f_{N-1}}, \bar{\tau}_{j+1 / 2}$ is biased in a mirrored fashion.

The parameter $\epsilon$ is a function of number of points in the discretization.

$$
\epsilon=\max \left(\left\|f_{0}\right\|,\left\|f_{0}^{\prime}\right\|\right)_{x \neq x_{d}}(\Delta \xi)^{3}, \quad \Delta \xi=\frac{1}{N},
$$

where $\left\|f_{0}\right\|$ and $\left\|f_{0}\right\|^{\prime}$ represent a norm of the flux and the gradient of the flux from the initial condition, where points near a discontinuity are excluded. ${ }^{\mathrm{A} 1}$

\footnotetext{
${ }^{\text {A1 }}$ For applications where the solution changes drastically during simulation, $\epsilon$ can be rescaled as necessary.
} 


\section{Appendix B}

\section{Recipe}

The recipe for calculating the gradient using the fourth-order ESWENO schemes is summarized below.

1. Construct the convective flux $\mathbf{f}$ at solution points $\left(\mathbf{x}_{N}\right)$ according to the governing differential equation(s).

2. For systems of equations (e.g. Euler), construct the flux Jacobian matrices: $A=\frac{\partial F}{\partial U}$ at the flux points $\left(\overline{\mathbf{x}}_{M}\right)$ using Roe average variables formed from nearest neighbor solution point data. Form the eigenvector decomposition $A=S \Lambda S^{-1}$.

3. At the flux point $\bar{x}_{j+\frac{1}{2}}$, identify all solution points $\mathbf{x}_{N}$ that contribute to the interpolants $M_{N} \mathcal{I}_{N}^{(r)}, r=L, C, R$. Use the eigenvector matrix $S_{j+\frac{1}{2}}^{-1}$ to rotate the solution and flux at these points, into characteristic form $C=S^{-1} U ; F_{c}(C)=$ $S^{-1} F(U)$.

4. Form the Lax-Friedrichs characteristic fluxes $\mathbf{f}_{\mathbf{c}}{ }^{ \pm}=\frac{1}{2}\left(\mathbf{f}_{\mathbf{c}} \pm \lambda_{\max } \mathbf{c}\right)$.

5. Perform interpolations on each candidate stencil, $\overline{\mathbf{f}}^{(r)}={ }_{M} \mathcal{I}_{N}^{(r)} \mathbf{f}_{\mathbf{c}}$.

6. Calculate the stencil biasing parameters:

(a) Calculate $\bar{\beta}^{r}$ for each flux point according to Eq. (A1), except at the endpoints.

(b) Calculate $\bar{\tau}_{j+1 / 2}$ according to Eq. (A3) and Eq. (A4).

7. Calculate and normalize the weights using the stencil biasing parameters and the target weights in Eq. (14).

8. Calculate $\Lambda_{i}$ from the weights using Eqs. (C3), (C4), and (C5) for the diagonalnorm scheme or Eqs. (D5) and (D6) for the block-norm.

9. Modify the diagonal of $\Lambda_{i}$ to be smoothly positive according to Eq. (20).

10. Calculate the WENO flux from the weights and candidate interpolations using Eq. (13).

11. Calculate the Energy Stable flux, $\bar{\psi}$, from Eq. (23).

12. Reconstruct the fluxes in characteristic space, $\overline{\mathbf{f}}_{j+1 / 2}=\overline{\mathbf{f}}_{j+1 / 2}^{+}+\overline{\mathbf{f}}_{j+1 / 2}^{-}$and $\bar{\psi}_{j+1 / 2}=\bar{\psi}_{j+1 / 2}^{+}+\bar{\psi}_{j+1 / 2}^{-}$, and transform back to physical space.

13. Calculate the gradient using the inverse of the P-norm, as in Eq. (24). 
Remark. It is important to note that all equations presented are for the forward propagating waves, $\bar{f}_{j+1 / 2}^{+}$. The equations for interpolation of backward propagating waves $\left(\bar{f}_{j+1 / 2}^{-}\right)$are found simply by flipping $r=(L L, L, C, R, R R)$ about $C$. For the $\Lambda_{i}$ terms, after flipping $r$ about $C$, the diagonal matrix is multiplied by -1 . Eq. (23) is also multiplied by -1 to calculate $\bar{\psi}_{j+1 / 2}^{-}$. 


\section{Appendix C}

\section{Fourth-order Diagonal-Norm Operator: $D_{2-4-2}$}

\section{C.1 Differentiation Matrix}

The target SBP differentiation operator $D_{2-4-2}$ can be written in the form

$D=\frac{1}{\Delta x}\left(\begin{array}{ccccccccccc}-\frac{24}{17} & \frac{59}{34} & -\frac{4}{17} & -\frac{3}{34} & 0 & 0 & 0 & 0 & 0 & 0 & 0 \\ -\frac{1}{2} & 0 & \frac{1}{2} & 0 & 0 & 0 & 0 & 0 & 0 & 0 & 0 \\ \frac{4}{43} & -\frac{59}{86} & 0 & \frac{59}{86} & -\frac{4}{43} & 0 & 0 & 0 & 0 & 0 & 0 \\ \frac{3}{98} & 0 & -\frac{59}{98} & 0 & \frac{32}{49} & -\frac{4}{49} & 0 & 0 & 0 & 0 & 0 \\ 0 & 0 & \frac{1}{12} & -\frac{2}{3} & 0 & \frac{2}{3} & -\frac{1}{12} & 0 & 0 & 0 & 0 \\ 0 & 0 & 0 & \ddots & \ddots & \ddots & \ddots & \ddots & 0 & 0 & 0 \\ 0 & 0 & 0 & 0 & \frac{1}{12} & -\frac{2}{3} & 0 & \frac{2}{3} & -\frac{1}{12} & 0 & 0 \\ 0 & 0 & 0 & 0 & 0 & \frac{4}{49} & -\frac{32}{49} & 0 & \frac{59}{98} & 0 & -\frac{3}{98} \\ 0 & 0 & 0 & 0 & 0 & 0 & \frac{4}{43} & -\frac{59}{86} & 0 & \frac{59}{86} & -\frac{4}{43} \\ 0 & 0 & 0 & 0 & 0 & 0 & 0 & 0 & -\frac{1}{2} & 0 & \frac{1}{2} \\ 0 & 0 & 0 & 0 & 0 & 0 & 0 & \frac{3}{34} & \frac{4}{17} & -\frac{59}{34} & \frac{24}{17}\end{array}\right)$

where the diagonal mass matrix $\mathcal{P}$ is

$$
P=\Delta x \operatorname{Diag}\left(\begin{array}{lllllllllll}
\frac{17}{48} & \frac{59}{48} & \frac{43}{48} & \frac{49}{48} & 1 & \ldots & 1 & \frac{49}{48} & \frac{43}{48} & \frac{59}{48} & \frac{17}{48}
\end{array}\right)
$$

The skew-symmetric matrix "Q" follows immediately from $P D$. 


\section{C.2 Interpolation Operators}

The WENO/ESWENO $2-4-2$ requires the inclusion of two extra stencils near the boundary. The interpolation coefficients are presented below.

$$
\begin{aligned}
& I_{L L}=\left(\begin{array}{cc}
0 & 0 \\
0 & 0 \\
0 & 0 \\
-\frac{71}{48} & \frac{119}{48} \\
0 & 0 \\
\vdots & \vdots \\
0 & 0 \\
-\frac{73}{48} & \frac{121}{48} \\
-\frac{17}{12} & \frac{29}{12} \\
-\frac{79}{48} & \frac{127}{48} \\
0 & 0
\end{array}\right) I_{L}=\left(\begin{array}{cc}
0 & 0 \\
0 & 0 \\
-\frac{7}{12} & \frac{19}{12} \\
-\frac{23}{48} & \frac{71}{48} \\
-\frac{1}{2} & \frac{3}{2} \\
\vdots & \vdots \\
-\frac{1}{2} & \frac{3}{2} \\
-\frac{25}{48} & \frac{73}{48} \\
-\frac{5}{12} & \frac{17}{12} \\
-\frac{31}{48} & \frac{79}{48} \\
0 & 0
\end{array}\right) I_{C}=\left(\begin{array}{cc}
1 & 0 \\
\frac{31}{48} & \frac{17}{48} \\
\frac{5}{12} & \frac{7}{12} \\
\frac{25}{48} & \frac{23}{48} \\
\frac{1}{2} & \frac{1}{2} \\
\vdots & \vdots \\
\frac{1}{2} & \frac{1}{2} \\
\frac{23}{48} & \frac{25}{48} \\
\frac{7}{12} & \frac{5}{12} \\
\frac{17}{48} & \frac{31}{48} \\
0 & 1
\end{array}\right) \\
& I_{R}=\left(\begin{array}{cc}
0 & 0 \\
\frac{79}{48} & -\frac{31}{48} \\
\frac{17}{12} & -\frac{5}{12} \\
\frac{73}{48} & -\frac{25}{48} \\
\frac{3}{2} & -\frac{1}{2} \\
\vdots & \vdots \\
\frac{3}{2} & -\frac{3}{2} \\
\frac{71}{48} & -\frac{23}{48} \\
\frac{19}{12} & -\frac{7}{12} \\
0 & 0 \\
0 & 0
\end{array}\right) I_{R R}=\left(\begin{array}{cc}
0 & 0 \\
\frac{127}{48} & -\frac{79}{48} \\
\frac{29}{12} & -\frac{17}{12} \\
\frac{121}{48} & -\frac{73}{48} \\
0 & 0 \\
\vdots & \vdots \\
0 & 0 \\
\frac{119}{48} & -\frac{71}{48} \\
0 & 0 \\
0 & 0 \\
0 & 0
\end{array}\right)
\end{aligned}
$$

\section{C.3 Target Weights}

The target weights for the five interpolation stencils are:

$$
\mathbf{d}_{242}=\left(\begin{array}{ccccc}
0 & 0 & 1 & 0 & 0 \\
0 & 0 & \frac{24}{31} & \frac{1013}{4898} & \frac{3}{158} \\
0 & \frac{11}{56} & \frac{51}{70} & \frac{3}{40} & 0 \\
\frac{3}{142} & \frac{357}{3266} & \frac{408}{575} & \frac{4}{25} & 0 \\
0 & \frac{1}{6} & \frac{2}{3} & \frac{1}{6} & 0 \\
\vdots & \vdots & \vdots & \vdots & \vdots \\
0 & \frac{1}{6} & \frac{2}{3} & \frac{1}{6} & 0 \\
0 & \frac{4}{25} & \frac{408}{575} & \frac{357}{3266} & \frac{3}{142} \\
0 & \frac{3}{40} & \frac{51}{70} & \frac{11}{56} & 0 \\
\frac{3}{158} & \frac{1013}{4898} & \frac{24}{31} & 0 & 0 \\
0 & 0 & 1 & 0 & 0
\end{array}\right)
$$




\section{C.4 Energy Stable Terms}

The nonzero terms of the diagonal matrices, $\Lambda_{i}$, for the $\mathrm{ESWENO}_{2-4-2}$ are

$\operatorname{Diag}\left(\Lambda_{1}\right)=\left(\begin{array}{c}0 \\ \frac{1}{96}\left(14-28 w_{2}^{(L)}-71 w_{3}^{(L L)}-31 w_{1}^{(R)}-31 w_{1}^{(R R)}\right) \\ \frac{1}{96}\left(-8+28 w_{2}^{(L)}-23 w_{3}^{(L)}+48 w_{3}^{(L L)}+31 w_{1}^{(R)}-20 w_{2}^{(R)}-48 w_{1}^{(R R)}\right) \\ \frac{1}{96}\left(2+23 w_{3}^{(L)}-24 w_{4}^{(L)}+23 w_{3}^{(L L)}+20 w_{2}^{(R)}-25 w_{3}^{(R)}+79 w_{1}^{(R R)}\right) \\ \frac{1}{96}\left(24 w_{4}^{(L)}-24 w_{5}^{(L)}+25 w_{3}^{(R)}-24 w_{4}^{(R)}\right) \\ \vdots \\ \frac{1}{4}\left(w_{i}^{(L)}-w_{i+1}^{(L)}+w_{i-1}^{(R)}-w_{i}^{(R)}\right) \\ \vdots \\ \frac{1}{96}\left(24 w_{N-4}^{(L)}-25 w_{N-3}^{(L)}+24 w_{N-5}^{(R)}-24 w_{N-4}^{(R)}\right) \\ \frac{1}{96}\left(-2+25 w_{N-3}^{(L)}-79 w_{N-1}^{(L L)}-20 w_{N-2}^{(L)}+24 w_{N-4}^{(R)}-23 w_{N-3}^{(R)}-23 w_{N-3}^{(R R)}\right. \\ \frac{1}{96}\left(8-31 w_{N-1}^{(L)}+48 w_{N-1}^{(L L)}+20 w_{N-2}^{(L)}+23 w_{N-3}^{(R)}-48 w_{N-3}^{(R R)}-28 w_{N-2}^{(R)}\right) \\ \frac{1}{96}\left(-14+31 w_{N-1}^{(L)}+31 w_{N-1}^{(L L)}+71 w_{N-3}^{(R R)}+28 w_{N-2}^{(R)}\right) \\ 0\end{array}\right.$

$\operatorname{Diag}\left(\Lambda_{2}\right)=\left(\begin{array}{c}0 \\ \frac{1}{96}\left(28 w_{2}^{(L)}+142 w_{3}^{(L L)}-31 w_{1}^{(R)}-110 w_{1}^{(R R)}\right) \\ \frac{1}{96}\left(23 w_{3}^{(L)}+94 w_{3}^{(L L)}-20 w_{2}^{(R)}-158 w_{1}^{(R R)}\right) \\ \frac{1}{96}\left(24 w_{4}^{(L)}-25 w_{3}^{(R)}\right) \\ \vdots \\ \frac{1}{4}\left(w_{i}^{(L)}-w_{i-1}^{(R)}\right) \\ \vdots \\ \frac{1}{96}\left(25 w_{N-3}^{(L)}-24 w_{N-4}^{(R)}\right) \\ \frac{1}{96}\left(158 w_{N-1}^{(L L)}+20 w_{N-2}^{(L)}-23 w_{N-3}^{(R)}-94 w_{N-3}^{(R R)}\right) \\ \frac{1}{96}\left(31 w_{N-1}^{(L)}+110 w_{N-1}^{(L L)}-142 w_{N-3}^{(R R)}-28 w_{N-2}^{(R)}\right. \\ 0\end{array}\right)$ 


$$
\operatorname{Diag}\left(\Lambda_{3}\right)=\left(\begin{array}{c}
0 \\
0 \\
\frac{1}{96}\left(-71 w_{3}^{(L L)}+79 w_{1}^{(R R)}\right) \\
\vdots \\
0 \\
\vdots \\
\frac{1}{96}\left(-79 w_{N-1}^{(L L)}+71 w_{N-3}^{(R R)}\right) \\
0 \\
0
\end{array}\right)
$$

The matrix Diag $\left(\Lambda_{0}\right)$ is identically zero. 


\section{Appendix D}

\section{Fourth-order Block-Norm Operator: $D_{3-4-3}$}

\section{D.1 Differentiation Matrix}

The target SBP differentiation operator $D_{3-4-3}$ satisfies the expression $D_{3-4-3}=$ $P_{3-4-3}^{-1} Q_{3-4-3}$ [see equation (10)]. The inverse P-norm for the $\mathrm{ESWENO}_{3-4-3}$ scheme is given by

$$
P_{3-4-3}^{-1}=\frac{1}{\Delta x}\left(\begin{array}{ccc}
P_{0}^{-1} & 0 & 0 \\
0 & I & 0 \\
0 & 0 & \left(P_{0}^{-1}\right)^{P T}
\end{array}\right) ; \quad P_{0}^{-1}=\left(\begin{array}{cccc}
\frac{71043003}{15979414} & -\frac{7964853}{15979414} & \frac{4820919}{1597414} & \frac{2199519}{15979414} \\
-\frac{7964853}{15979414} & \frac{14093655}{15979414} & \frac{331299}{1597414} & -\frac{1445721}{15979414} \\
\frac{4820919}{15979414} & \frac{331299}{15979414} & \frac{18932367}{15923414} & \frac{253203}{15979414} \\
\frac{2199519}{15979414} & -\frac{1457521}{15979414} & \frac{253203}{15979414} & \frac{15938451}{15979414}
\end{array}\right)
$$

where $P T$ denotes the per-symmetric transpose. The $Q_{3-4-3}$ matrix is given by

$$
Q_{3-4-3}=\left(\begin{array}{ccccccccccc}
-\frac{1}{2} & \frac{101}{144} & -\frac{29}{144} & 0 & 0 & 0 & 0 & 0 & 0 & 0 & 0 \\
-\frac{101}{144} & 0 & \frac{17}{24} & -\frac{1}{144} & 0 & 0 & 0 & 0 & 0 & 0 & 0 \\
\frac{29}{144} & -\frac{17}{24} & 0 & \frac{85}{144} & -\frac{1}{12} & 0 & 0 & 0 & 0 & 0 & 0 \\
0 & \frac{1}{144} & -\frac{85}{144} & 0 & \frac{2}{3} & -\frac{1}{12} & 0 & 0 & 0 & 0 & 0 \\
0 & 0 & \frac{1}{12} & -\frac{2}{3} & 0 & \frac{2}{3} & -\frac{1}{12} & 0 & 0 & 0 & 0 \\
0 & 0 & 0 & \ddots & \ddots & \ddots & \ddots & \ddots & 0 & 0 & 0 \\
0 & 0 & 0 & 0 & \frac{1}{12} & -\frac{2}{3} & 0 & \frac{2}{3} & -\frac{1}{12} & 0 & 0 \\
0 & 0 & 0 & 0 & 0 & \frac{1}{12} & -\frac{2}{3} & 0 & \frac{85}{144} & -\frac{1}{144} & 0 \\
0 & 0 & 0 & 0 & 0 & 0 & \frac{1}{12} & -\frac{85}{144} & 0 & \frac{17}{24} & -\frac{29}{144} \\
0 & 0 & 0 & 0 & 0 & 0 & 0 & \frac{1}{144} & -\frac{17}{24} & 0 & \frac{101}{144} \\
0 & 0 & 0 & 0 & 0 & 0 & 0 & 0 & \frac{29}{144} & -\frac{101}{144} & \frac{1}{2}
\end{array}\right)
$$

(D2) 


\section{D.2 Interpolation Operators}

The interpolation coefficients for the $\mathrm{ESWENO}_{3-4-3}$ are

$$
I_{L}=\left(\begin{array}{cc}
0 & 0 \\
0 & 0 \\
-\frac{25}{36} & \frac{61}{36} \\
-\frac{61}{144} & \frac{205}{144} \\
-\frac{1}{2} & \frac{3}{2} \\
\ddots & \ddots \\
-\frac{1}{2} & \frac{3}{2} \\
-\frac{83}{144} & \frac{227}{144} \\
-\frac{11}{36} & \frac{47}{36} \\
-\frac{101}{144} & \frac{245}{144} \\
0 & 0
\end{array}\right) I_{C}=\left(\begin{array}{cc}
1 & 0 \\
\frac{101}{144} & \frac{43}{144} \\
\frac{11}{36} & \frac{25}{36} \\
\frac{83}{144} & \frac{61}{144} \\
\frac{1}{2} & \frac{1}{2} \\
\ddots & \ddots \\
\frac{1}{2} & \frac{1}{2} \\
\frac{61}{144} & \frac{83}{144} \\
\frac{25}{36} & \frac{11}{36} \\
\frac{43}{144} & \frac{101}{144} \\
0 & 1
\end{array}\right) I_{R}=\left(\begin{array}{cc}
0 & 0 \\
\frac{245}{144} & -\frac{101}{144} \\
\frac{47}{36} & -\frac{11}{36} \\
\frac{227}{144} & -\frac{83}{144} \\
\frac{3}{2} & -\frac{1}{2} \\
\ddots & \ddots \\
\frac{3}{2} & -\frac{1}{2} \\
\frac{205}{144} & -\frac{61}{144} \\
\frac{61}{36} & -\frac{25}{36} \\
0 & 0 \\
0 & 0
\end{array}\right)
$$

\section{D.3 Target Weights}

The target weights for each stencil in $\mathrm{ESWENO}_{3-4-3}$ are given below for each flux point.

$$
\mathbf{d}_{343}=\left(\begin{array}{ccc}
0 & 1 & 0 \\
0 & \frac{72}{101} & \frac{29}{101} \\
\frac{29}{100} & \frac{189}{275} & \frac{1}{44} \\
\frac{1}{61} & \frac{4248}{5063} & \frac{12}{83} \\
\frac{1}{6} & \frac{2}{3} & \frac{1}{6} \\
\vdots & \vdots & \vdots \\
\frac{1}{6} & \frac{2}{3} & \frac{1}{6} \\
\frac{12}{83} & \frac{4248}{5063} & \frac{1}{61} \\
\frac{1}{44} & \frac{189}{275} & \frac{29}{100} \\
\frac{29}{101} & \frac{72}{101} & 0 \\
0 & 1 & 0
\end{array}\right)
$$




\section{D.4 Energy Stable Terms}

The nonzero terms of the diagonal matrices, $\Lambda_{i}$, for the $\mathrm{ESWENO}_{3-4-3}$ are

$$
\begin{aligned}
& \operatorname{Diag}\left(\Lambda_{1}\right)=\left(\begin{array}{c}
0 \\
\frac{1}{288}\left(58-100 w_{2}^{(L)}-101 w_{1}^{(R)}\right) \\
\frac{1}{288}\left(-56+100 w_{2}^{(L)}-61 w_{3}^{(L)}+101 w_{1}^{(R)}-44 w_{2}^{(R)}\right) \\
\frac{1}{288}\left(22+61 w_{3}^{(L)}-72 w_{4}^{(L)}+44 w_{2}^{(R)}-83 w_{3}^{(R)}\right) \\
\frac{1}{288}\left(72 w_{4}^{(L)}-72 w_{5}^{(L)}+83 w_{3}^{(R)}-72 w_{4}^{(R)}\right) \\
\vdots \\
\frac{1}{4}\left(w_{i}^{(L)}-w_{i+1}^{(L)}+w_{i-1}^{(R)}-w_{i}^{(R)}\right) \\
\vdots \\
\frac{1}{288}\left(72 w_{N-4}^{(L)}-83 w_{N-3}^{(L)}+72 w_{N-5}^{(R)}-72 w_{N-4}^{(R)}\right) \\
\frac{1}{288}\left(-22+83 w_{N-3}^{(L)}-44 w_{N-2}^{(L)}+72 w_{N-4}^{(R)}-61 w_{N-3}^{(R)}\right) \\
\frac{1}{288}\left(56-101 w_{N-1}^{(L)}+44 w_{N-2}^{(L)}+61 w_{N-3}^{(R)}-100 w_{N-2}^{(R)}\right.
\end{array}\right) \\
& \operatorname{Diag}\left(\Lambda_{2}\right)=\left(\begin{array}{c}
0 \\
\frac{1}{288}\left(100 w_{2}^{(L)}-101 w_{1}^{(R)}\right) \\
\frac{1}{288}\left(61 w_{3}^{(L)}-44 w_{2}^{(R)}\right) \\
\frac{1}{288}\left(72 w_{4}^{(L)}-83 w_{3}^{(R)}\right) \\
\vdots \\
\frac{1}{4}\left(w_{i}^{(L)}-w_{i-1}^{(R)}\right) \\
\vdots \\
\frac{1}{288}\left(83 w_{N-3}^{(L)}-72 w_{N-4}^{(R)}\right) \\
\frac{1}{288}\left(44 w_{N-2}^{(L)}-61 w_{N-3}^{(R)}\right) \\
\frac{1}{288}\left(101 w_{N-1}^{(L)}-100 w_{N-2}^{(R)}\right) \\
0
\end{array}\right)
\end{aligned}
$$




\section{Appendix E}

\section{References}

1. Erlebacher, G.; Hussaini, M.; and Shu, C.-W.: Interaction of a shock with a longitudinal vortex. Journal of Fluid Mechanics, vol. 337, 1997, pp. 129-153.

2. Zhang, S.; Jiang, S.; Zhang, Y.; and Shu, C.: The mechanism of sound generation in the interaction between a shock wave and two counter-rotating vortices. Physics of Fluids, vol. 21(7), 2009.

3. Shen, Y.; Wang, B.; and Zha, G.: Implicit WENO Scheme and High Order Viscous Formulas for Compressible Flows. 25th AIAA Applied Aerodynamics Conference, no. 2007-4431, 2007.

4. Cockburn, B.; Johnson, C.; Shu, C.-W.; and Tadmor, E.: Advanced Numerical Approximation of Nonlinear Hyperbolic Equations. Springer-Verlag, 1998.

5. Yamaleev, N. K.; and Carpenter, M. H.: Third-order Energy stable WENO Scheme. Journal of Computational Physics, vol. 228, 2009, pp. 3025-3047.

6. Yamaleev, N. K.; and Carpenter, M. H.: A systematic methodology for constructing high-order energy stable WENO schemes. Journal of Computational Physics, vol. 228, 2009, pp. 4248-4272.

7. Jiang, G.; and Shu, C.-W.: Efficient implementation of weighted ENO schemes. Journal of Computational Physics, vol. 126, 1996, pp. 202-228.

8. Martin, M.; Taylor, E.; Wu, M.; and Weris, V.: A bandwidth-optimized WENO scheme for the effective direct numerical simulation of compressible turbulence. Journal of Computational Physics, vol. 220, 2006, pp. 270-289.

9. Kreiss, H.; and Scherer, G.: Mathematical Aspects of Finite Elements in Partial Differential Equations, Academic Press, New York, Finite element and finite difference methods for hyperbolic partial differential equations. 1974.

10. Carpenter, M. H.; Nordström, J.; and Gottlieb, D.: A stable and conservative interface treatment of arbitrary spatial accuracy. Journal of Computational Physics, vol. 148, 1999, pp. 341-365.

11. Strand, B.: Summation by parts for finite difference approximations for $\mathrm{d} / \mathrm{dx}$. Journal of Computational Physics, vol. 110(1), 1994, pp. 47-67.

12. Nordström, J.: Conservative finite difference formulations, variable coefficients, energy estimates and artificial dissipation. Journal of Scientific Computing, vol. 29, 2006, pp. 375-404. 
13. Kitson, A.; McLachlan, R. I.; and Robidoux, N.: Skew-adjoint finite difference methods on nonuniform grids. New Zealand Journal of Mathematics, vol. 32, no. 2, 2003, pp. 139-159.

14. McLachlan, R. I.; and Robidoux, N.: Antisymmetry, pseudospectral methods, and conservative PDEs, World Scientific, EQUADIFF 99, B. Fiedler, K. Groger, and J. Sprekels, eds. 2000, pp. 994-999.

15. Gustafsson, B.: The convergence rate for difference approximations to mixed initial boundary value problems. Mathematics of Computation, vol. 29, 1975, pp. 396-406.

16. Svärd, M.; and Nordström, J.: On the order of accuracy for difference approximations of initial-boundary value problems. Journal of Computational Physics, vol. 218, 2006, pp. 333-352.

17. http://www.wolfram.com/products/mathematica/index.html, 2009.

18. Carpenter, M. H.; and Kennedy, C. A.: Fourth-Order 2N-Storage Runge-Kutta Schemes. TM 109112, NASA, 1994.

19. Kim, J. W.: Optimized boundary compact finite difference schemes for computational aeroacoustics. Journal of Computational Physics, vol. 225, 2007, pp. 995-1019.

20. Carpenter, M. H.; Gottlieb, D.; and Abarbanel, S.: Time-Stable Boundary Conditions for Finite-Difference Schemes Solving Hyperbolic Systems: Methodology and Application to High-Order Compact Schemes. Journal of Computational Physics, vol. 111, 1994, pp. 220-236.

21. Svärd, M.; Carpenter, M. H.; and Nordström, J.: A stable high-order finite difference scheme for the compressible Navier-Stokes equations, far-field boundary conditions. Journal of Computational Physics, vol. 225, 2007, pp. 1020-1038.

22. http://www.cantera.org, 2009. 


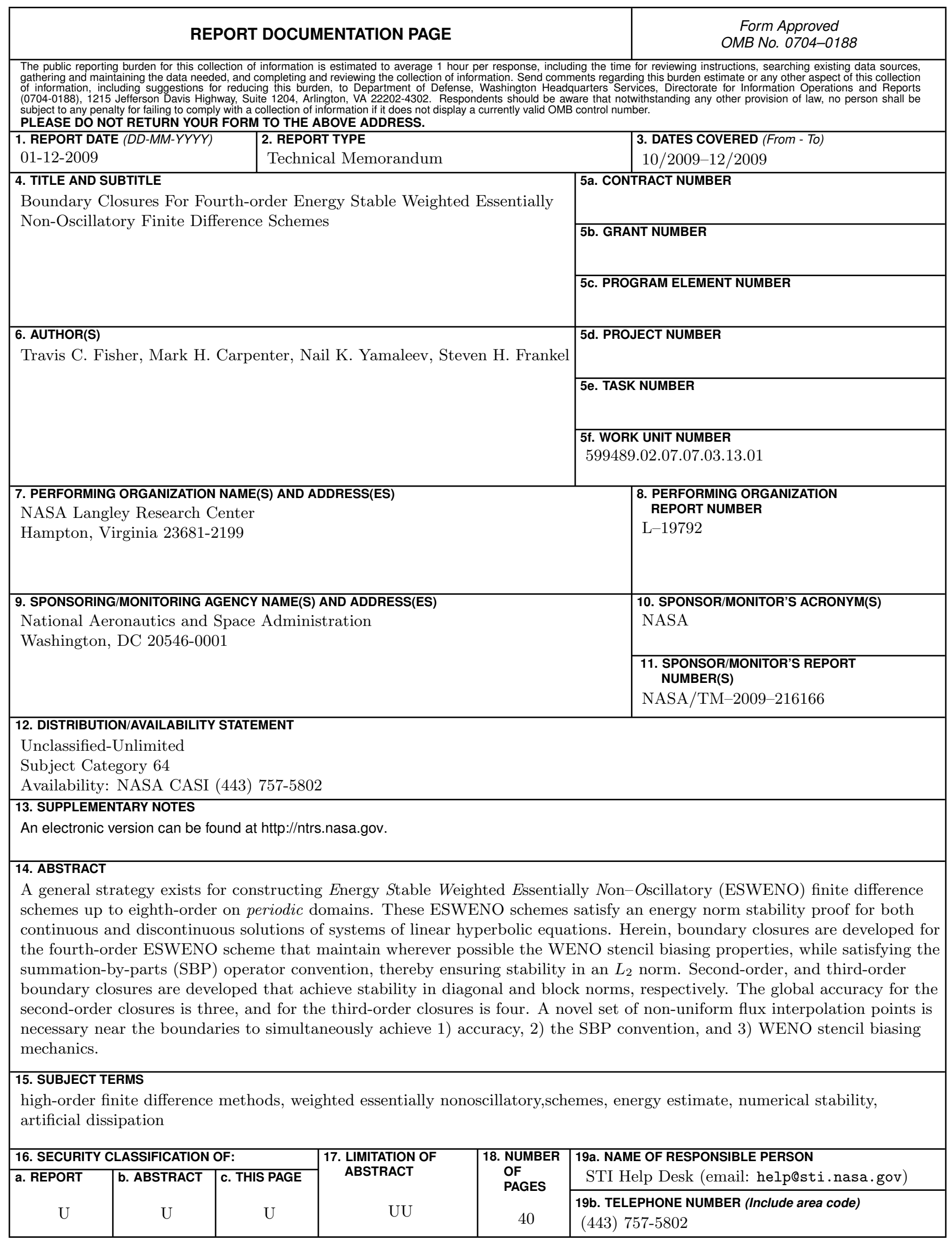





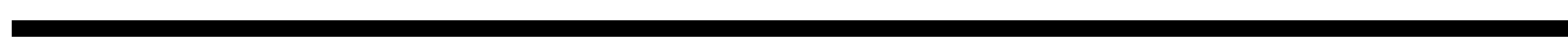

